\title{
A neural network for online spike classification that improves decoding accuracy
}

\author{
Deepa Issar ${ }^{1,2}$, Ryan C. Williamson ${ }^{2,3,5}$, Sanjeev B. Khanna ${ }^{1}$, and Matthew A. Smith ${ }^{4,5,6 \#}$ \\ ${ }^{1}$ Department of Bioengineering, University of Pittsburgh, Pittsburgh, PA, USA \\ ${ }^{2}$ University of Pittsburgh School of Medicine, University of Pittsburgh, Pittsburgh, PA, USA \\ ${ }^{3}$ Department of Machine Learning, Carnegie Mellon University, Pittsburgh, PA, USA \\ ${ }^{4}$ Department of Biomedical Engineering, Carnegie Mellon University, Pittsburgh, PA, USA \\ ${ }^{5}$ Carnegie Mellon Neuroscience Institute, Pittsburgh, PA, USA \\ ${ }^{6}$ Department of Ophthalmology, University of Pittsburgh School of Medicine, University of Pittsburgh, \\ Pittsburgh, PA, USA \\ \#: Address correspondence to: \\ Matthew A. Smith \\ Carnegie Mellon University \\ Department of Biomedical Engineering and Neuroscience Institute \\ Email: matt@smithlab.net \\ Running head: A neural network for online spike classification
}


[A neural network for online spike classification] 1 stability. However, most algorithms for separating neural action potentials from noise are not suitable for use in real time and have shown mixed effects on decoding performance. With the goal of removing noise that impedes online decoding, we sought to automate the intuition of human spike-sorters to operate in real time with an easily tunable parameter governing the stringency with which spike waveforms are classified. We trained an artificial neural network with one hidden layer on neural waveforms that were hand-labeled as either spikes or noise. The network output was a likelihood metric for each waveform it classified, and we tuned the network's stringency by varying the minimum likelihood value for a waveform to be considered a spike. Using the network's labels to exclude noise waveforms, we decoded remembered target location during a memory-guided saccade task from electrode arrays implanted in prefrontal cortex of rhesus macaque monkeys. The network classified waveforms in real time, and its classifications were qualitatively similar to those of a human spike-sorter. Compared to decoding with threshold crossings, in most sessions we improved decoding performance by removing waveforms with low spike likelihood values. Furthermore, decoding with our network's classifications became more beneficial as time since array implantation increased. Our classifier serves as a feasible preprocessing step, with little risk of harm, that could be applied to both offline neural data analyses and online decoding.

While there are many spike-sorting methods that isolate well-defined single units, these methods typically New \& Noteworthy involve human intervention and have inconsistent effects on decoding. We used human classified neural waveforms as training data to create an artificial neural network that could be tuned to separate spikes from noise that impaired decoding. We found that this network operated in real time and was suitable for both offline data processing and online decoding. 


\section{Introduction}

Brain computer interfaces (BCIs) have been used as both research tools to understand neural

29 phenomena (Chase, Kass, and Schwartz 2012; Golub, Yu, and Chase 2015; Sadtler et al. 2014; Schafer

30 and Moore 2011) and devices to improve patient control of prosthetics (Collinger et al. 2013; Hochberg et

31 al. 2012; Velliste et al. 2008). BCIs interpret neural signals arising from electrodes implanted in the

32 cortex using real time decoding algorithms; however, their performance is limited by the difficulty of

33 isolating the activity of individual neurons from extracellular voltage signals ("spike-sorting"), the

34 characteristics of the information present in individual neurons and multi-unit activity (their "tuning"), as

35 well as the nuances of interactions among neurons (Averbeck, Latham, and Pouget 2006). Refining the

36 raw data is necessary to improve BCI performance, but identifying waveforms that contain relevant

37 information is challenging.

38 One common noise removal method is to use all waveforms crossing a minimum voltage

39 threshold for decoding, where all waveforms on a particular channel are considered to be from a single

40 neural "unit" (Christie et al. 2015; Lewicki 1998). Using threshold crossings also serves to reduce the

41 amount of stored data. However, threshold crossings often capture noise and the summed activity of

42 multiple nearby neurons, called multi-unit activity, on each channel (Rey, Pedreira, and Quian Quiroga

43 2015; Stark and Abeles 2007). Alternatively, since waveforms with the characteristic shape of an action

44 potential are the focus of most offline neural data analyses, a different approach is to isolate well-defined

45 spike waveforms from the threshold crossings via spike-sorting, and use only those waveforms for

46 decoding (Lewicki 1998; Rey, Pedreira, and Quian Quiroga 2015). Unfortunately, most spike-sorting

47 techniques are unsuitable for BCI use because they are time-intensive and require manual refinement.

48 Furthermore, even with the development of real time, automated spike-sorting algorithms

49 (Chaure, Rey, and Quian Quiroga 2018; Chung et al. 2017), deciding which waveforms should be used by

50 the decoding algorithm is challenging. There is typically no clear ground truth for what constitutes a spike

51 waveform based solely on sparse extracellular recordings (Pedreira et al. 2012; Rey, Pedreira, and Quian

52 Quiroga 2015; Rossant et al. 2016; Wood et al. 2004), except in the case of a few rare data sets 
53 (Anastassiou et al. 2015; Neto et al. 2016). While some studies have found spike-sorted units improved 54 decoding performance (Kloosterman et al. 2014; Santhanam et al. 2004; Todorova et al. 2014; Ventura 55 and Todorova 2015), others have found that using pooled spikes from threshold crossings resulted in 56 comparable or better decoding that was more stable over time (Chestek et al. 2011; Christie et al. 2015; 57 Dai et al. 2019; Fraser et al. 2009; Gilja et al. 2012). These conflicting results highlight the gap in our 58 understanding of what information in neural recordings is most valuable for BCIs. Even within studies, 59 there are inconsistent effects of spike-sorting on decoding, both between subjects and over time (Christie 60 et al. 2015; Fraser et al. 2009). It is also difficult to compare the effects of spike-sorting on decoding 61 performance across studies due to the inherent variability in spike-sorting techniques (Pedreira et al.

62 2012; Rey, Pedreira, and Quian Quiroga 2015; Rossant et al. 2016; Wood et al. 2004) and differences in 63 brain regions, parameters being studied, and decoding algorithms that may influence which waveforms 64 are the most decodable (Bishop et al. 2014; Chestek et al. 2011; Fraser et al. 2009; Lewicki 1998; Oby et al. 2016). Furthermore, it is unclear whether spike-sorting, where the goal is to define isolated singleunits, is the best-suited approach for preprocessing data for decoding, where multi-unit activity has been shown to be sufficient in some cases (Stark and Abeles 2007). explicitly sorting our data and instead developed an easily tunable spike classifier that could objectively and efficiently assign waveforms to one of two classes: spike or noise. To achieve this goal, we sought a

71 classification approach that would (1) take advantage of the spike-sorted waveforms from previous

72 analyses that formed a readily available, labeled training data set; (2) output a likelihood of being a spike

73 for each waveform so we could simply adjust the minimum cutoff for binary spike classification; (3) once

74 trained could operate in real time. We built a neural network classifier that satisfied these three criteria.

75 To evaluate our classifier's performance, we decoded the activity of sparse electrode arrays implanted in

76 prefrontal cortex (PFC), and compared decoding accuracy of task condition with all threshold crossings to

77 decoding accuracy with the waveforms selected by our classifier. We also assessed how decoding

78 performance changed as a function of the stringency of our classifier (i.e. the minimum cutoff for spike 
79 classification) and explored how decoding accuracy with our classifier changed as a function of time

80 since the array implantation (array implant age).

81 By removing waveforms that the network identified as unlikely to be spikes, we improved

82 decoding performance for most sessions relative to decoding with all threshold crossings. Moreover, in

83 the remaining sessions there was no substantial detriment to decoding accuracy, meaning we could apply

84 our method with little risk of harming decoding performance. Our network classifications continued to

85 improve decoding accuracy in sessions recorded long after array implantation, even though the overall

86 decoding accuracy decreased and the array recordings became noisier. Thus, our real time, tunable

87 waveform classifier demonstrates promise for long-term BCI applications and for efficient offline

88 preprocessing.

\section{Materials and Methods}

91 We trained a neural network, using a database of spike-sorted waveforms, to assign waveforms to

92 a spike or noise class. We then tested the network's classifications with another data set that was

93 independent from the training set. In contrast to the offline, human supervised spike-sorting that was used

94 to label the training data, our network did not sort waveforms into isolated single-units, but rather

95 assigned spike or noise classifications to individual waveforms (hence its name Not A Sorter, or NAS). To

96 evaluate the network's classifications, we decoded task location using only waveforms labeled as spikes

97 by the network and compared the accuracy to decoding accuracy using all threshold crossings. All

98 experimental procedures were approved by the Institutional Animal Care and Use Committee of the

99 University of Pittsburgh and were performed in accordance with the United States National Institutes of

100 Health's Guidelines for the Care and Use of Laboratory Animals.

$101 \quad$ Neural recordings

102 We analyzed neural recordings from six adult male rhesus macaques (Macaca mulatta) which

103 had previously been spike-sorted for ongoing experiments in the laboratory. Data from four subjects were

104 used to train our spike-classifying neural network, and data from the remaining two subjects (Monkey Pe 
105 and Monkey Wa) were used to assess decoding accuracy using the network's classifications. Raw

106 recordings from both 96-electrode 'Utah' arrays (Blackrock Microsystems, Salt Lake City, UT) and 16-

107 channel linear microelectrode arrays (U-Probe, Plexon, Dallas, TX) were band-pass filtered from 0.3 to

$1087,500 \mathrm{~Hz}$, digitized at $30 \mathrm{kHz}$, and amplified by a Grapevine system (Ripple, Salt Lake City, UT). The

109 inter-electrode distance on the 'Utah' arrays was $400 \mu \mathrm{m}$ and on the linear arrays was 150 or $200 \mu \mathrm{m}$,

110 beyond the range over which the two extracellular electrodes are likely to capture the waveform of a

111 single neuron. For each recording session, a threshold $\left(\mathrm{V}_{\mathrm{T}}\right)$ was defined for each channel independently

112 based on the root-mean-squared voltage of the waveforms $\left(\mathrm{V}_{\mathrm{RMS}}\right)$ recorded on that channel at the

113 beginning of the session (i.e. $\mathrm{V}_{\mathrm{T}}=\mathrm{V}_{\mathrm{RMS}} * \mathrm{X}$, where $\mathrm{X}$ was a multiplier set by the experimenter). Each time

114 the signal crossed that threshold, a 52-sample waveform segment was captured (i.e. a threshold crossing),

115 with 15 samples prior to and 36 samples following the sample in which the threshold excursion occurred.

\section{Offline spike-sorting}

117 All data in this work were spike-sorted to identify well-defined single-units for previous studies.

118 This pre-sorted data was used to create the labeled training set for this study. Waveform segments were

119 initially sorted into spike units and noise using a custom, offline MATLAB spike-sorting algorithm that

120 used an automated competitive mixture decomposition method (Shoham, Fellows, and Normann 2003).

121 These automated classifications for each recording session were subsequently refined manually by a

122 researcher using custom MATLAB software (https://github.com/smithlabvision/spikesort). The researcher

123 modified the classifications on each channel based on visualization of the overlaid waveform clusters,

124 projections of the waveforms in PCA space (to assess how many clusters were present), the inter-spike

125 interval distribution of any potential single-unit (ensuring it contained few waveforms separated by less

126 than a sensible refractory period), and whether each potential single-unit was stationary (i.e. present for

127 the majority of the recording session). While only a single researcher spike-sorted the waveforms from

128 any particular session, the data in the training set ( 7 sessions total) collectively consisted of data spike-

129 sorted by four different researchers. If multiple unique spike waveform shapes were present on a channel,

130 the sorter would label those as different units. 
We sought a diverse neural waveform training set that captured a variety of subjects, brain

133 regions, implanted array ages (i.e. number of days since the array was implanted) and recording devices

134 (Table 1). The four training data set subjects each had a 96-electrode 'Utah' array implanted in right or

135 left hemisphere visual area V4. Two of those subjects also had 16-channel U-probe recordings from the

136 frontal eye field (FEF), located in the anterior bank of the arcuate sulcus. Data from two sessions were

137 used for three of the subjects: one session recorded shortly after the array implant, and one session

138 recorded at least four months later. For the fourth subject, only a single recording session recorded shortly

139 after the array implant was used because there were no later recordings from that array. These recordings

140 were collected and sorted as part of previous studies which describe the experimental preparations in

141 detail (Khanna, Snyder, and Smith 2019; Snyder et al. 2014; Snyder et al. 2015; Snyder and Smith 2015).

142 Data in the training set were assigned a label of 0 or 1 . If there were multiple single-units identified by the

143 sorter on a particular channel, they were not distinguished in the training set and all were treated as spike

144 waveforms (labeled as 1). We only included data from channels with very distinct spike waveforms,

145 defined as channels with a signal-to-noise ratio (SNR) greater than 2.5 (Kelly et al. 2007). These channels

146 still included both noise (labeled as 0 ) and spike waveforms (labeled as 1). The aim of using only the high

147 SNR channels in the training set was to emphasize relatively well-isolated single-unit action potential

148 shapes while also exposing the network to a variety of noise waveforms. Additionally, excluding low

149 SNR channels resulted in a training set with a relatively even distribution of spike and noise waveforms

$150 \quad$ (47.5\% spikes, $52.5 \%$ noise) .

151 Overall, the network training set consisted of 24,810,795 waveforms from four monkeys, two

152 brain regions (V4 and FEF), two recording devices (Utah array and U-probe), and different array implant

153 ages. These waveforms were classified as spikes or noise using the offline spike-sorting technique

154 described above (Fig. 1a).

Table 1. Training data set information, including time since implant (array age), brain area (RHright hemisphere, LH-left hemisphere), and number of recording sessions. Utah arrays contained 96 channels and U-Probes had 16 channels. 


\begin{tabular}{lllll}
\hline \multirow{3}{*}{ Monkey Wi } & Recording device & Brain area & Time since implant & \# of sessions \\
\cline { 2 - 5 } & Utah array & RH V4 & $\sim 1$ month & 1 \\
\cline { 2 - 5 } & Utah array & RH V4 & $\sim 11$ months & 1 \\
\cline { 2 - 5 } U-Probe & RH FEF & - & 1 \\
\hline \multirow{2}{*}{ Monkey Ro } & Utah array & LH V4 & $<1$ month & 1 \\
\cline { 2 - 5 } & Utah array & LH V4 & $\sim 8$ months & 1 \\
\cline { 2 - 5 } & U-Probe & LH FEF & - & 1 \\
\hline \multirow{2}{*}{ Monkey Bo } & Utah array & RH V4 & $<1$ month & 1 \\
\cline { 2 - 5 } & Utah array & RH V4 & $\sim 4$ months & 1 \\
\hline Monkey Bu & Utah array & RH V4 & $\sim 1$ month & 1 \\
\hline
\end{tabular}

\section{Testing data set}

157 We used recordings from two additional subjects, not included in the training set, for testing to

158 ensure that the trained network was generalizable to subjects not included in its training. The two testing

159 data set subjects (Monkey Pe and Monkey Wa) each had two 96-electrode 'Utah' arrays implanted, one in

160 visual area V4 and one in dorsolateral PFC (on the prearcuate gyrus just medial to the principal sulcus,

161 area 8Ar). Both arrays were implanted in the right hemisphere for Monkey Pe and in the left hemisphere

162 for Monkey Wa. Only data recorded from PFC were used for the complete set of analyses (Table 2)

163 because this provided a matched data set from two monkeys for the purpose of assessing the impact of our

164 method on decoding and because it allowed us to test our network's generalizability in situations where

165 the data were recorded from a different region than the training set. However, we repeated some of the

166 analyses in held out data from V4 and similarly found an improvement in decoding with our network

167 (Supplementary Figure 5, https://doi.org/10.6084/m9.figshare.11808492.v1). Recordings within 50 days

168 of the array implant were considered early sessions and recordings from over 50 days after the implant

169 were considered late sessions (see Table 2 for session counts by subject). For Monkey Pe, the thresholds

170 across channels were similar for all recording sessions $(90 \%$ of channels had thresholds between -39 and -

$17120 \mu \mathrm{V}$, median: $-29 \mu \mathrm{V}$ ). For Monkey Wa, the thresholds were more permissive for the later recording

172 sessions ( $>6$ months after the array implant) as the experimenters sought to extract the maximum

173 remaining signal in arrays that were decreasing in recording quality. The median threshold of Monkey

174 Wa's early sessions was $-32 \mu \mathrm{V}(90 \%$ between -43 and $-23 \mu \mathrm{V})$, and the median threshold of the later

175 sessions was $-15 \mu \mathrm{V}(90 \%$ between -29 and $-12 \mu \mathrm{V})$. The waveforms in the testing data set were also 
176 spike-sorted offline via the technique described above for a comparative analysis between our network 177 classifications and offline spike-sorting.

Table 2. Testing data set information, including time since implant (array age), brain area (RHright hemisphere, LH-left hemisphere), and number of recording sessions.

\begin{tabular}{lllll}
\hline & Recording device & Brain area & Time since implant & \# of sessions \\
\hline \multirow{2}{*}{ Monkey Pe } & Utah array & RH PFC & $11-43$ days & 14 \\
\cline { 2 - 5 } & & RH PFC & $80-147$ days & 22 \\
\hline \multirow{2}{*}{ Monkey Wa } & Utah array & LH PFC & $27-40$ days & 5 \\
\cline { 2 - 5 } & & LH PFC & $180-224$ days & 11 \\
\hline
\end{tabular}

Neural Network

181 to classify data segments as spike or noise waveforms (Fig. 1b). The network accepts a waveform

182 segment with ' $s$ ' samples ( $s=52)$, which passes through a hidden layer with ' $n$ ' units $(n=50)$ that uses a

183 rectified linear unit (ReLU) activation function. The product of the hidden layer passes through one

184 output unit and then the network applies a sigmoid function to its output.

With the aforementioned training waveforms, the network was trained to maximize accuracy 2015) and a binary cross-entropy loss function in batch sizes of 100 .

For a single waveform input run through the trained network, the output was a value between 0

189 (likely not a spike) and 1 (likely a spike), which represented the network's assessment of the likelihood

190 that the input waveform was a spike. While this output value was not a conventional probability, we refer

191 to it as the probability of being a spike or $\mathrm{P}($ spike) because it was a likelihood metric scaled between 0

192 and 1. Supplementary Figure 1 (https://doi.org/10.6084/m9.figshare.11808492.v1) provides more

193 intuition regarding the hidden layer units and how the network assessed waveforms. In order to classify

194 the waveforms using the network's output probability, we set a minimum $\mathrm{P}$ (spike) for a waveform to be

195 classified as a spike. We referred to this minimum probability as the $\gamma$ threshold. A $\gamma$ threshold of 0

196 classified all of the waveforms that were captured for the session as spikes; this is often referred to as 
198 because the $\mathrm{P}($ spike) cutoff was higher.

We refer to our neural network as Not A Sorter (NAS), because it classifies waveforms as spikes

201 scripts used to train the neural network and classify waveforms, as well as sample training and testing

202 data, are available at https://doi.org/10.6084/m9.figshare.11808492.v1. The NAS software is also

203 integrated into our custom MATLAB “Spikesort” package (https://github.com/smithlabvision/spikesort).

\section{Memory-guided saccade task}

205 The two testing data set subjects (Monkey Pe and Monkey Wa) performed a memory guided 206 saccade task (Fig. 2a). Each subject fixated on a central point for $200 \mathrm{~ms}$. Then, a target stimulus flashed 207 at a set amplitude and direction for $50 \mathrm{~ms}$, followed by a $500 \mathrm{~ms}$ delay. There were a total of 40 possible 208 conditions: the radius could be one of five eccentricities and the target direction could be one of eight 209 (spaced in $45^{\circ}$ steps). After the delay, the fixation point disappeared instructing the subject to make a 210 saccade to the location where the target flashed. For some sessions, during the saccade the target

211 reappeared to help the subject; however, for all analyses we only used data from the beginning of the

212 delay period (i.e. prior to the saccade). Subjects were rewarded with water or juice for making a saccade

213 to the correctly remembered target location. For all decoding analyses, the decoded condition was the

214 target location. Trials at a single eccentricity (Monkey Pe: $9.9^{\circ}$, Monkey Wa: $\sim 7.6^{\circ}$ ) were used to

215 maximize the number of recording sessions that could be compared. Thus, there were eight unique target

216 locations to decode.

217 Only rewarded trials were analyzed. The number of trial repeats per condition varied over

218 sessions. Monkey Pe had an average of 43 repeats per session (range: 25-75, sd: 17) and Monkey Wa had 219 an average of 62 repeats per session (range: 51-83, sd: 11) for each target condition.

\section{Decoding}

221 To classify target direction prior to movement from spikes in PFC, we used a Poisson Naïve

222 Bayesian decoder (Fig. 2b). All decoding analyses were performed offline. Waveforms classified as 
[A neural network for online spike classification] 10

223 spikes from $300 \mathrm{~ms}$ to $500 \mathrm{~ms}$ after fixation (i.e. $50 \mathrm{~ms}$ after stimulus offset) were used for decoding,

224 while waveforms classified as noise were discarded. Each channel was considered a single decodable unit

225 with one exception: to calculate decoding accuracy using manual spike-sorting, if there were multiple

226 single-units identified by the manual spike-sorting method on a channel, each single-unit on that channel

227 remained separate for the purposes of decoding. Units with an average firing rate across trials below 0.25

228 spikes per second were discarded. We allocated training and test trials using two different methods. For

229 one analysis, we assigned $80 \%$ of the trials from each session to the training set and the remaining $20 \%$ to

230 the test set (Fig. 4a,b). For the remaining analyses, we wanted two test sets, so we used 60\% of the trials

231 for training and $20 \%$ for each of the two test sets. The two test sets allowed us to search for an optimal $\gamma$

232 threshold using the first test set and cross validate this value with the second test set. Training and test

233 data were rotated such that each trial was used for a test set only once.

234 Our decoding algorithm created a Poisson distribution model for each target location $(\theta)$ using

235 the average spike count for each unit $\left(n_{\text {spike }}\right)$ in the training set. For each test trial, the target location

236 with the maximum prediction probability, $P\left(\theta \mid n_{\text {spike }}\right)$, was the predicted label. In equation (1),

$237 P\left(n_{\text {spike }} \mid \theta\right)$ was calculated using the Poisson model developed with the training trials:

$$
P\left(\theta \mid n_{\text {spike }}\right)=\frac{P\left(n_{\text {spike }} \mid \theta\right) * P(\theta)}{P\left(n_{\text {spike }}\right)}
$$

total number of predictions. We used 5-fold cross validation and computed the average decoding accuracy

240 across folds.

\section{Results}

We trained a one-layer neural network called Not a Sorter (NAS) to evaluate the likelihood that a

244 neural waveform was a spike. We used a diverse set of waveforms in the training set from different brain

245 regions, subjects, and array implant ages (time since array implant) in order to expose the network to a

246 variety of waveform types. Each waveform in the training set was assigned a binary label of noise (0) or 
[A neural network for online spike classification] 11

247 spike (1) via offline spike-sorting with manual refinement by researchers. The network was also exposed

248 to variability in spike classification due to manual sorting because different researchers sorted different

249 sets of waveforms in the training data. The network learned to assess how spike-like a waveform was

250 based on binary labels, but itself output a continuous value between 0 and 1 for each waveform allowing

251 for tunable classification. We referred to this output as the probability of being a spike or P(spike). The

252 network classified 1000 waveforms in less than one millisecond on average (computed on a 2011 iMac

253 with a $2.8 \mathrm{GHz}$ Intel Core 17 processor). In a $10 \mathrm{~ms}$ bin, 1000 waveforms would be the expected output of

2541000 channels each recording from a single-unit with a firing rate of $100 \mathrm{spikes} / \mathrm{s}$. Thus, our network

255 could be easily integrated to operate within real time computing constraints. In a realistic simulation of a

256 real time application of our network, waveforms captured from 192 channels in a 20 ms time step could

257 be classified in less than $0.1 \mathrm{~ms}$ on average, easily sufficient for the updating of a BCI cursor or other

258 feedback.

\section{Qualitative assessment of NAS classifications}

260 To assign each NAS-classified waveform a binary spike or noise label, we set a parameter called

261 the $\gamma$ threshold, which was the minimum P(spike) a waveform needed to be considered a spike waveform.

262 We found that even a low $\gamma$ threshold, such as $\gamma=0.20$ (Fig 3a), assigned most waveforms to classes that a

263 human spike-sorter would deem appropriate. Most spike-sorters and spike-sorting algorithms search for

264 waveforms with a canonical action potential shape - an initial voltage decrease followed by a sharp

265 increase, a narrow peak, and a return to baseline. Increasing the $\gamma$ threshold to 0.70 (i.e. only waveforms

266 assigned a $\mathrm{P}($ spike $)>0.70$ were considered spikes) mimicked the effect of more selective spike-

267 classification (Fig 3b), where the percentage of spike waveforms on the channel decreased since more

268 waveforms were placed in the noise class and the remaining spike waveforms had a clearer single-unit

269 shape. Considering all of the waveforms from this same channel, the range of $\mathrm{P}($ spike) values coincided

270 well with our subjective impression of the match of individual waves with a canonical action potential

271 shape (Fig 3c), and this was also true across all channels from this array (Fig 3d). Thus, tuning the $\gamma$ 
[A neural network for online spike classification] 12

272 threshold from low values (near 0) to high values (near 1) resulted in a shift from a more permissive to a 273 more restrictive regime.

\section{Quantitative assessment of NAS classifications with decoding}

275 Although by eye the network appeared to classify waveforms reasonably well, we sought to

276 objectively assess its performance by measuring its effect on offline decoding accuracy. Specifically, we

277 hypothesized that decoding using spikes classified by our network would be better than using threshold

278 crossings. We applied our network to a set of data recorded from PFC on which the network had not been

279 trained. First, we set the $\gamma$ threshold and discarded any waveforms with a P(spike) below the threshold.

280 Next, we split the data into training and testing sets. We trained the decoder using the training set to

281 decode the task condition (the remembered location, out of 8 possibilities) during the delay period of a

282 memory-guided saccade task on each trial (see Methods). Then, we used the trained decoder to assess

283 decoding accuracy in the test set(s). We repeated this process for the same data set using multiple $\gamma$

284 thresholds between 0 (i.e., all threshold crossings were considered spikes) and 0.95 (i.e., only waveforms

285 assigned a 0.95 or greater probability by the network were considered spikes). Since only waveforms

286 classified as spikes were used for decoding, as the $\gamma$ threshold increased, fewer waveforms remained for

287 decoding.

Initially, we used $80 \%$ of trials to train the decoder and the remaining to test it. We analyzed

289 cross-validated decoding accuracy as a function of $\gamma$ (sample recording sessions in Fig. 4a,b). Chance

290 decoding was $12.5 \%$ (1 out of 8) and was verified by computing decoding accuracy for shuffled test trials.

291 The decoding accuracy using threshold crossings $(\gamma=0)$ for the sample recording sessions in Fig. 4a and

$2924 \mathrm{~b}$ was close to chance levels for Monkey Pe (12.8\%) and was also low for Monkey Wa (20.1\%). Using

293 the network's classifications improved decoding accuracy relative to using threshold crossings. The peak

294 decoding benefit of using the network's spike classifications occurred at a low $\gamma$ threshold (Monkey Pe: $\gamma$

$295=0.16$, Monkey Wa: $\gamma=0.16$ ). Being very selective about the spikes used for decoding (by setting a high

$296 \gamma$ threshold) did not have a large impact on decoding accuracy, which appeared to plateau after the peak.

297 We found that increments of the $\gamma$ threshold did not remove the same number of waveforms. A substantial 
[A neural network for online spike classification] 13

298 proportion of waveforms were removed at the lowest $\gamma$ threshold tested (especially in Monkey Pe), and 299 small increments of the $\gamma$ threshold as it approached a value of 1 could also result in large increases in the 300 proportion of removed waveforms (especially notable in Figure 4b). For reference, when we manually 301 spike-sorted the same data offline (see Methods), we discarded 89.2\% of the waveforms in Figure 4a and $30271.5 \%$ of the waveforms in Figure 4b. These values were similar to the percentage of waveforms the 303 network removed at higher $\gamma$ thresholds.

304 Ideally, for the sake of simplicity during online BCI experiments, we could select a single $\gamma$ 305 threshold and use it for all recording sessions. We wanted to find the lowest $\gamma$ threshold that improved 306 decoding accuracy for the majority of recording sessions. We focused on lower $\gamma$ thresholds because it 307 was a conservative approach, and because our intuition from single channels and results in example 308 sessions (Figure 4) showed it could have the greatest benefit for decoding. In order to cross-validate both 309 our decoder and our $\gamma$ threshold selection for the remaining analyses we created two test sets for decoding 310 (training set: $60 \%$ of trials, test set $1: 20 \%$ of trials, test set $2: 20 \%$ of trials). Our general strategy was to 311 use the first test set to identify a $\gamma$ threshold that optimized decoding accuracy. We then cross-validated 312 this selection by applying the chosen $\gamma$ threshold to the data in the second test set and computing decoding 313 accuracy for those unseen trials.

314 As described above, after training the decoder for each session using the training trials, we 315 calculated decoding accuracy with the first set of test trials at each $\gamma$ threshold. We found the maximum 316 decoding accuracy at any $\gamma$ threshold greater than 0 , and then searched for the lowest non-zero $\gamma$ threshold 317 that resulted in a decoding accuracy within $99 \%$ of that maximum (Fig. 4c, d). Since the optimal $\gamma$ 318 threshold varied between sessions, we computed the median across sessions of these $\gamma$ values and rounded 319 it to the nearest tested value (Monkey Pe: $\gamma=0.2$, Monkey Wa: $\gamma=0.08$ ). We then used that median 320 value as the $\gamma$ threshold for all sessions in the second test set and analyzed the change in decoding 321 accuracy from decoding using threshold crossings in that same test set, which we termed $\Delta \%$ decoding 322 accuracy (Fig. 4e, f). Combining sessions from both animals, the average improvement in decoding 323 accuracy was 3.9\% (2-tailed, Wilcoxon signed rank test; across subjects: $p<0.0001$, Monkey Pe: $p<$ 
[A neural network for online spike classification] 14

324 0.0001, Monkey Wa: $p=0.057)$. Thus, our network's classifications tuned to the previously described $\gamma$

325 thresholds resulted in a net benefit for decoding performance across sessions compared to using threshold 326 crossings. Although we could tune the $\gamma$ threshold for each session to maximize the decoding, our choice

327 of a fixed $\gamma$ threshold was more consistent with the use of our network in an online decoding context,

328 where it would be desirable to set a constant $\gamma$ threshold at the start of each session rather than tuning it as

329 a free parameter. However, an alternative strategy would be to collect a small data set at the start of each

330 day to find the optimal $\gamma$ value, and then continue experiments for the remainder of that day using the

331 chosen value.

332 Given the ability of our network to improve decoding accuracy beyond that observed with

333 threshold crossings, we took advantage of our longitudinal recordings in a fixed paradigm to understand

334 how our network's performance varied as a function of time. Since there is mixed evidence in the

335 literature regarding decoding stability with long-term array implants, we were specifically interested in

336 how the passage of time since array implant (array implant age), and concomitant degradation of

337 recording quality, could influence our network's performance.

338 Impact of array age

339 First, we assessed how the overall quality of our neural data changed as a function of time. As the

340 time since the array implant increased, the percentage of waveforms that the network assigned a very low

341 probability of being a spike $(\mathrm{P}($ spike $)<0.02)$ also increased (Fig 5a; Spearman's correlation, Monkey Pe:

$342 \rho=0.87, p<0.0001$; Monkey Wa: $\rho=0.90, p<0.0001$ ). Conversely, the percentage of waveforms assigned

343 higher probabilities of being a spike $(\mathrm{P}($ spike $)>0.70)$ decreased over time (Fig 5b; Monkey Pe: $\rho=-0.87$,

$344 p<0.0001$; Monkey Wa: $\rho=-0.94, p<0.0001)$. These classification changes were consistent with our

345 qualitative observations that the arrays showed an increasing proportion of multi-unit activity (relative to

346 single-unit activity) and apparent noise over time. These changes were also reflected in our manual spike-

347 sorting (performed offline on the same data for previous studies, see Methods) where we discarded a

348 smaller percentage of waveforms from earlier sessions that were 0-50 days post-implant (mean \%

349 waveforms removed +/- 1 sd, Monkey Pe: 36.1 +/- 8.2\%; Monkey Wa: 46.1 +/- 12.2\%) and a larger 
[A neural network for online spike classification] 15

350 percentage in later sessions that were greater than 50 days post-implant (mean \% waveforms removed +/-

$3511 \mathrm{sd}$, Monkey Pe: 87.0 +/- 7.7\%; Monkey Wa: 77.8 +/- 10.3\%). We further found that the ratio of the

352 percentage of waveforms assigned a high $\mathrm{P}($ spike) to the percentage of waveforms assigned a low

$353 \mathrm{P}($ spike) by the network served as a proxy for a signal to noise ratio metric, and it was highly correlated

354 with the median signal to noise ratio (across channels) of the array in each session (Supplementary Figure

355 2, https://doi.org/10.6084/m9.figshare.11808492.v1). Both signal to noise metrics decreased over time,

356 and the decoding accuracy with threshold crossings also decreased the longer the array had been

357 implanted (Fig 5c; Monkey Pe: $\rho=-0.86, p<0.0001$; Monkey Wa: $\rho=-0.69, p=0.004$ ).

358 Given the change in distribution of the types of waveforms present during the session over time,

359 we tried to optimize the $\gamma$ threshold based on time since array implant. We used the same method as in

360 Fig. 4c-d to compute the $\gamma$ thresholds with approximately maximum decoding (within 1\%), except we

361 calculated the median of the early sessions (recorded 0-50 days post implant) and the median of the late

362 sessions (recorded $>50$ days post implant) separately. This did not prove to be a useful optimization as the

363 median values were similar between early and late sessions in both Monkey Pe $\left(\gamma_{\text {early }}=0.25, \gamma_{\text {late }}=0.18\right)$

364 and Monkey Wa $\left(\gamma_{\text {early }}=0.06, \gamma_{\text {late }}=0.08\right)$. Decoding accuracy compared to threshold crossings $(\Delta \%$

365 decoding accuracy) was significantly increased in Monkey Pe (Figure 5d, 2-tailed, Wilcoxon signed rank

366 test, $p<0.0001)$ and was not significantly helped or hurt in Monkey Wa $(\mathrm{p}=0.09)$. In line with the trends

367 in signal and noise over time, the decoding accuracies from later sessions ( $>50$ days post implant) were

368 helped more by the network classifications in both subjects than those from earlier sessions (Figure 5d,

369 Wilcoxon rank sum, Monkey Pe: $p=0.01$; Monkey Wa: $p=0.01$ ). When we normalized the decoding

370 accuracy at each $\gamma$ threshold by the decoding accuracy using threshold crossings for each session and

371 separately averaged across early and late sessions, the increased benefit of using the network in the later

372 sessions compared to the earlier sessions was clear (Figure 5e).

373 Altogether, these results provide additional evidence that factors such as time since the array was

374 implanted and signal to noise ratio influence decoding accuracy and affect how noise removal impacts

375 decoding performance. Despite decreasing signal quality and increasing noise waveforms, the effect of 
[A neural network for online spike classification] 16

376 using our network classifications for noise removal prior to decoding was consistent in that it was most

377 often beneficial for decoding and at worst minimally detrimental. We confirmed that these results were

378 not affected by poor $\gamma$ threshold selection by using the maximum decoding accuracy regardless of $\gamma$

379 threshold to calculate $\Delta \%$ decoding accuracy (Supplementary Figure 3a,

380 https://doi.org/10.6084/m9.figshare.11808492.v1). Our results were also not substantially influenced by

381 variability in the number of trials across sessions (Supplementary Figure 3b,

382 https://doi.org/10.6084/m9.figshare.11808492.v1). An even simpler alternative to our network might be

383 to adjust the voltage threshold for capturing waveforms, to make it more or less permissive. We found

384 that increasing this threshold (making it more negative and therefore excluding the waveforms that did

385 not exceed it) did not improve decoding performance (Supplementary Figure 4,

$386 \mathrm{https} / /$ doi.org/10.6084/m9.figshare.11808492.v1), indicating that our network was not merely acting to

387 exclude small amplitude waveforms. Lastly, we confirmed that our network was beneficial for decoding

388 in data recorded from another brain region (V4) with different Utah arrays in the same sessions from the

389 same animals in the test data set (Supplementary Figure 5,

390 https://doi.org/10.6084/m9.figshare.11808492.v1).

391 Comparison to spike-sorting

392 In light of the inconsistent effect of spike-sorting on decoding performance in the literature

393 (Christie et al. 2015; Dai et al. 2019; Fraser et al. 2009; Todorova et al. 2014), we sought to evaluate the

394 relative merits of manual spike-sorting and our network classifier on decoding accuracy in our data. Our

395 goal was to place our new method (using our network to remove noise but not sort the data) in context of

396 previous work that evaluated the impact of human supervised spike-sorting on decoding. We took

397 advantage of the offline spike-sorting that had already been applied to these data and calculated the

398 decoding accuracy of spike-sorted data (Fig. 6, data aggregated across subjects in the marginal

399 histograms). Decoding using spike-sorted waveforms was better than using threshold crossings (Fig. 6

400 right marginal; 2-tailed, Wilcoxon signed rank test, $p<0.0001)$ similar to decoding with our network

401 classifications (Fig. 6 top marginal, data from Fig. 5d aggregated across subjects; $p<0.0001$ ). Our 
[A neural network for online spike classification] 17

402

403

404

405

406

407

408

409

410 separate spikes from noise in extracellular electrophysiological recordings in real time. To assess the value of this method, we used an objective criterion - the ability to decode remembered location from prefrontal cortex in a memory-guided saccade task. We found that we could set a fixed $\gamma$ threshold that resulted in an improvement or at worst a small change in decoding accuracy for the majority of sessions, and this effect persisted even as recording quality decreased over time. Compared to spike-sorting (performed offline with substantial manual labor involved), classifying spikes with our network had similar effects on decoding accuracy relative to threshold crossings and was less tedious.

\section{Training the network}

Developing a neural network trained on spike-sorted data allowed us to capture the essence of the rules by which experienced researchers distinguish spikes from noise without explicitly defining all the variables and exceptions, a standard challenge in spike classification (Wood et al. 2004). Several studies have demonstrated the promise of using a neural network trained on spike-sorted or human-verified data for spike detection and classification (Chandra and Optican 1997; Kim and Kim 2000; Lee et al. 2017;

427 Racz et al. 2019; Saif-Ur-Rehman et al. 2019). Lee et al. (2017), Racz et al. (2019), and Saif-Ur-Rehman 
[A neural network for online spike classification] 18

428 et al. (2019) used spike-sorted data to train their respective neural networks and developed more complex

429 network architectures than the one-layer network we explored in this work. Each of these studies was

430 validated by the performance of the network in correctly labeling held out spiking waveforms. The

431 intention of our simple architecture was not to compare its performance to existing spike detection and

432 sorting algorithms, but rather to explore how a relatively simple, fast, and trainable noise removal method

433 could improve decoding performance.

434 We found that a simple neural network was quite good at capturing the nuances of the training

435 set. Experts often disagree on how to classify certain instances of low SNR waveforms. The network was

436 sensitive enough to capture a researcher's specific spike classification tendencies, such that a network

437 trained on data classified by a particular individual performed better on classifying test data from that

438 individual than from other expert spike classifiers. Given this level of sensitivity, a reasonable concern

439 would be the presence of misclassifications in our training set, as human spike-sorting is both subjective

440 and prone to error (Pedreira et al. 2012; Rossant et al. 2016). However, the network was trained on many

441 waveforms to minimize the impact of any confounders, including waveforms from multiple subjects,

442 brain regions, and human spike-sorters. Additionally, by training the network on labeled data with some

443 variability introduced by human sorters, the network learned to make stronger predictions (higher

$444 \mathrm{P}($ spike) values) for obvious spike waveforms and weaker predictions for more ambiguous waveforms.

445 We then leveraged this range of prediction values to create a tunable parameter for our classifications (i.e.

446 the $\gamma$ threshold).

An ideal and even more generalizable training set might include waveforms from different

448 research groups, artificially simulated neural waveforms and noise in which ground truth could be

449 established, and/or pre-defined quantities of certain types of spikes (e.g. shifted spikes, slow and fast

450 spike waveforms) and noise (e.g. electrical artifacts) to target what the network learns. However, no

451 matter how much training data is used, an ideal training set could only be defined in the context of

452 objective metrics to assess the trained network's performance.

\section{$453 \quad$ Evaluating the network with decoding}


[A neural network for online spike classification] 19

We compared decoding performance with our network classifications to performance with

threshold crossings, which are the current standard in the BCI community. We used independent test data

456 to assess the effect of classifications from our trained network on decoding accuracy. Although the

457 expectation might be that removing noise from neural data would improve decoding, historically doing so

458 with spike-sorted classifications has had mixed effects, in some cases hurting performance (Todorova et

459 al. 2014). Nevertheless, many BCIs currently operate with some amount of online noise removal or visual

460 inspection (such as disabling visibly noisy channels) to preprocess neural data before decoding (Chase,

461 Kass, and Schwartz 2012; Hochberg et al. 2006; Homer et al. 2013; Sadtler et al. 2014). The question of

462 whether to spike-sort BCI data prior to decoding or simply use all threshold crossings has been a source

463 of debate with no clear resolution. Our work reframes this question to investigate how an automated noise

464 removal method could be used to aid decoding.

Our findings provide additional support, across multiple sessions over time, that removing certain

noise waveforms prior to decoding provides an advantage over using all threshold crossings, consistent

467 with findings from Fraser et al. (2009) and Christie et al. (2015). Although there were a few occasions

when decoding accuracy with threshold crossings was better than decoding using the network, it was

471 array on certain days would limit the benefits of our network and thereby increase the relative likelihood

472 that waveforms valuable for decoding were removed.

Contrary to the benefits of spike-classification that we observed, a study by Todorova et al.

474 (2014) found that using spike-sorted waveforms hurt decoding when noise waveforms were discarded. It

475 is difficult to speculate why removing noise waveforms hurt decoding in their study but helped it in ours

476 given that our methods of noise removal were different. They also found assigning noise waveforms from

477 spike-sorting to a new unit, rather than discarding them, improved decoding relative to threshold

478 crossings. Yet, when we assigned our noise waveforms to separate units, decoding accuracy was worse or

479 unchanged compared to discarding noise waveforms both with our spike-sorted classifications and our 
[A neural network for online spike classification] 20

network classifications (Supplementary Figure 6, https://doi.org/10.6084/m9.figshare.11808492.v1).

481 Importantly, we defined 'noise' operationally as any waveform that did not exceed the $\gamma$ threshold of our

482 network setting. This definition of noise surely included both electrical artifacts as well as indistinct

483 shapes that were actually neural in origin. Removal of waveforms from the former class could only help

484 decoding, but removal of the latter class might actually harm decoding if the multi-unit activity contained

485 in those waveforms had information about the condition of interest for decoding.

486 Apparent noise in neuronal activity, in addition to changing the activity of single electrode

487 channels, can also be correlated across channels. In the activity of pairs of single neurons, such

488 fluctuations in trial-to-trial correlated variability are sometimes termed "noise correlation" (also known as

489 spike count correlation, or $r_{s c}$ ). Noise correlation can have a substantial impact on the ability of a decoder

490 to extract information from neuronal populations (Averbeck, Latham, and Pouget 2006; Kohn et al.

491 2016), and the activity of multi-unit groups of neurons has a higher noise correlation than that of the

492 constituent pairs of neurons (Cohen and Kohn 2011). Since our network acted on threshold crossings,

493 which typically contain the activity of many individual neurons, it likely also impacted the magnitude of

494 the noise correlation between channels. Our decoder assumed that the noise on each unit was

495 independent, a choice common to BCI, which permitted training the decoder with a smaller quantity of

496 trials than is necessary to learn the covariance structure in the population. The effect of our network on

497 decoding surely depends on not only the structure of the network and the choice of $\gamma$ threshold, but also

498 the structure of noise present in the population and the sensitivity of the decoder to that noise.

In addition to understanding how the type of noise present in the data impacts decoding, it may

500 also be useful to evaluate the types of neural waveforms that contribute to decoding. Different levels of

501 single and multi-unit activity are captured during a neural recording depending on the threshold set by the

502 researcher (i.e. the minimum voltage level at which the neural activity is marked as a spike). By adjusting

503 this threshold, we change the candidate waveforms available for decoding. Oby et al. (2016) found that

504 the optimal threshold depended on the type of information being extracted from neural activity. Another

505 study found that higher thresholds, which captured less multi-unit activity, resulted in worse decoding of 
[A neural network for online spike classification] 21

direction from M1 (Christie et al. 2015). These studies in the context of our own findings highlight the need to identify specific types of waveforms that contribute to the decodable information in different

508 brain regions and task contexts.

\section{Other variables that impact decoding accuracy}

A key concern for practical implementation of BCIs is decoding stability. Array recordings often

511 get visibly noisier over time, potentially as a result of an immune response to the implant (Ward et al.

512 2009) or a physical shift in position (Perge et al. 2013). In our study, as the time since array implant

513 increased there were more "low probability" waveforms and fewer "high probability" waveforms,

514 indicating the arrays had fewer well-defined single-unit waveforms. In line with this, several other studies

515 with chronic array implants have found that the number of single-units decreased over time (Dickey et al.

516 2009; Downey et al. 2018; Fraser and Schwartz 2012; Tolias et al. 2007). Nevertheless, there are mixed

517 findings on the stability of decoding performance in chronic array implants. Some studies have found

518 decoder performance with threshold crossings remained stable over time (Chestek et al. 2011; Flint et al.

519 2016; Gilja et al. 2012; Nuyujukian et al. 2014); however, other studies, including our own, observed a

520 decrease in decoding accuracy over time (Perge et al. 2014; Wang et al. 2014). Unlike the previous

521 literature investigating the effects of threshold crossings and spike-sorting on decoding performance, we

522 used neural activity from PFC, where decoding accuracy is typically lower (Boulay et al. 2016; Jia et al.

523 2017; Meyers et al. 2008; Parthasarathy et al. 2017; Rizzuto et al. 2005; Spaak et al. 2017; Tremblay et al.

524 2015) than in motor cortex (Collinger et al. 2013; Koralek et al. 2012; Masse et al. 2014; Sadtler et al.

525 2015). Stability of decoding accuracy with array age may also depend on the brain region of the implant,

526 the subject's task, initial decoding performance, and the choice to train the decoder daily (Chestek et al.

527 2011; Gilja et al. 2012) or hold it constant (Nuyujukian et al. 2014). The beneficial effects of our network

528 on decoding were particularly salient in the case of older array implants, where decoding accuracy had

529 decreased over time.

530 Limitations of $N A S$ 
[A neural network for online spike classification] 22

Our network, NAS, is certainly not sufficient to achieve the goal of obtaining well-isolated

532 single-units. NAS evaluates each waveform independently and therefore, cannot distinguish between

533 multiple units recorded on the same channel. A concern for online decoding is that pooling spikes on a

534 given channel could hurt decoding if there are multiple single-units that respond to the task conditions

535 differently. This did not appear to be a major issue in our data because we did not find a substantial

536 difference between decoding performance with pooled spikes from the network classifications and

537 decoding with well-isolated single-units from spike-sorting (Fig. 7).

$538 \quad$ NAS was trained using previously sorted data available in our laboratory, rather than simulated

539 spike waveforms and noise (Chaure, Rey, and Quian Quiroga 2018) or a ground truth data set

540 (Anastassiou et al. 2015; Neto et al. 2016). This choice had the potential to limit the abilities of NAS to

541 distinguish spikes from noise, as our training data was subject to errors and biases of individual human

542 sorters. However, given the rarity of such ground truth data sets, and the particular features of spikes that

543 are unique to brain areas and the recording hardware in a laboratory, we also view this choice as a

544 strength. Nearly any laboratory performing extracellular electrophysiology would have such training data

545 available, already suitable for their particular brain areas and recording methods. Thus our method is

546 highly customizable for any laboratory, and we view its focus on human-sorted training data as a strength

547 for the particular application to which it was designed.

548 Additionally, NAS does not take advantage of temporal or spatial information. When manually

549 spike-sorting, sorters often use the inter-spike interval and the stationarity of a candidate unit over the

550 recording to decide whether those waveforms could sensibly belong to a single-unit. For dense arrays and

551 tetrodes, leveraging spatial information is vital for isolating spikes that appear on multiple channels

552 (Chung et al. 2017; Pachitariu et al. 2016). However, such dense recordings are still rare relative to the

553 use of sparse electrodes in electrophysiological experiments. Thus, for traditional offline data processing,

554 NAS could be used as a quick preprocessing step to remove noise and make preliminary spike

555 classifications but must be accompanied by another algorithm or manual spike-sorting to isolate single-

556 units. 
[A neural network for online spike classification] 23

\section{Extensions for NAS}

Our neural network-based spike classifier is a promising tool for both offline preprocessing of neural data and improving online decoding performance. However, in designing our network we only scratched the surface of many potential avenues to address these challenges. We found that using networks with different numbers of hidden units and layers did not substantially alter decoding accuracy even though there were some differences in how these different sized networks classified waveforms. Given that our network was relatively simple, it would be possible to implement similar operations with alternative algorithms such as logistic regression. We chose a neural network because it was easily trainable from existing data and there are many ways to modularly build upon its complexity. Although we opted to use 50 hidden units and one hidden layer, it is possible that a more complex network with additional filtering operations and more categories of waveform classification may result in improved decoding performance and could help to create a more robust spike classifier with the ability to distinguish multi-units and single-units for offline analyses. However, our work had the value of exploring how a smaller network trained on spike-sorted data could identify features that were most valuable for decoding.

While training on spike-sorted data from channels with well-isolated single-unit action potentials $(\mathrm{SNR}>2.5)$ was an efficient choice because of its easy availability, it may not be the best choice in regimes where canonical spike-shapes do not necessarily carry the most decodable information compared to less well-defined multi-unit activity (Chestek et al. 2011; Stark and Abeles 2007). If the goal is to improve decoding performance, it might be valuable to train a network on waveforms from low SNR channels that contain more multi-unit waveforms or to select waveforms or units that positively contribute to decoding. Ventura and Todorova (2015) developed a method for identifying the information contribution of units for decoding. Such a method could be used to develop a training set of neural waveforms that are assigned a label based on how much they positively or negatively contributed to decoding. Alternatively, if the goal is to discard specific types of artifacts, then it might be ideal to train the network on predominantly low SNR channels that contain those artifacts. 
[A neural network for online spike classification] 24 neural data analyses. A recent study found that spike-sorting did not provide much benefit over threshold crossings for estimating neural state space trajectories (Trautmann et al. 2019), a powerful demonstration

586 that the principles of neural circuits may be accessible in neuronal population recordings even when 587 single units are not identified. However, this analysis was performed on trial-averaged data. Using NAS 588 classifications might be more helpful in single-trial data where estimating trajectories through neural state

589 space could be negatively influenced by the momentary entry of noise into recordings. Another

590 interesting application would be to assess how NAS classification impacts the measurement of neural

591 noise correlations, which have been shown to be affected by the stringency of spike-sorting (Cohen and

592 Kohn 2011). Using NAS classifications with a low $\gamma$ threshold could prove to be a useful noise removal 593 tool for applications that are sensitive to classification stringency.

Overall, we developed a new tool for preprocessing BCI data that classified threshold crossings in

595 a tunable manner that was beneficial for decoding. A neural network-based spike classifier has the 596 potential to reduce the need for human intervention in removing noise from neural data. Our tunable

597 classifier is a step toward preprocessing methods that both optimize and stabilize online decoding 598 performance.

\section{Acknowledgements}

602 We would like to thank Samantha Schmitt for assistance with data collection and Ben Cowley, Byron Yu, 603 and Steve Chase for their valuable insight on this project.

\section{Grants}

606 D.I. was supported by National Science Foundation (NSF) DBI 1659611, National Institutes of Health

607 (NIH) T32 GM008208, and the ARCS foundation Thomas-Pittsburgh Chapter Award. M.A.S. was

608 supported by NIH R01EY022928, R01MH118929, R01EB026953, and P30EY008098; NSF NCS 
[A neural network for online spike classification] 25

1734901; a career development grant and an unrestricted award from Research to Prevent Blindness; the

Eye and Ear Foundation of Pittsburgh. R.C.W. was supported by a Richard King Mellon

Foundation Presidential Fellowship in the Life Sciences. S.B.K. was supported by T32 EY017271.

\section{Disclosures}

The authors declare no conflicts of interest.

\section{References}

Abadi, Martín, Ashish Agarwal, Paul Barham, Eugene Brevdo, Zhifeng Chen, Craig Citro, Greg S. Corrado, Andy Davis, Jeffrey Dean, Matthieu Devin, Sanjay Ghemawat, Ian Goodfellow, Andrew Harp, Geoffrey Irving, Michael Isard, Yangqing Jia, Rafal Jozefowicz, Lukasz Kaiser, Manjunath Kudlur, Josh Levenberg, Dan Mane, Rajat Monga, Sherry Moore, Derek Murray, Chris Olah, Mike Schuster, Jonathon Shlens, Benoit Steiner, Ilya Sutskever, Kunal Talwar, Paul Tucker, Vincent Vanhoucke, Vijay Vasudevan, Fernanda Viegas, Oriol Vinyals, Pete Warden, Martin Wattenberg, Martin Wicke, Yuan Yu, and Xiaoqiang Zheng. 2016. 'TensorFlow: LargeScale Machine Learning on Heterogeneous Distributed Systems'.

Anastassiou, C. A., R. Perin, G. Buzsaki, H. Markram, and C. Koch. 2015. 'Cell type- and activitydependent extracellular correlates of intracellular spiking', J Neurophysiol, 114: 608-23.

Averbeck, B. B., P. E. Latham, and A. Pouget. 2006. 'Neural correlations, population coding and computation', Nat Rev Neurosci, 7: 358-66.

Bishop, W., C. C. Chestek, V. Gilja, P. Nuyujukian, J. D. Foster, S. I. Ryu, K. V. Shenoy, and B. M. Yu. 2014. 'Self-recalibrating classifiers for intracortical brain-computer interfaces', J Neural Eng, 11: 026001.

Boulay, C. B., F. Pieper, M. Leavitt, J. Martinez-Trujillo, and A. J. Sachs. 2016. 'Single-trial decoding of intended eye movement goals from lateral prefrontal cortex neural ensembles', J Neurophysiol, 115: 486-99.

Chandra, R., and L. M. Optican. 1997. 'Detection, classification, and superposition resolution of action potentials in multiunit single-channel recordings by an on-line real-time neural network', IEEE Trans Biomed Eng, 44: 403-12.

Chase, S. M., R. E. Kass, and A. B. Schwartz. 2012. 'Behavioral and neural correlates of visuomotor adaptation observed through a brain-computer interface in primary motor cortex', $J$ Neurophysiol, 108: 624-44.

Chaure, F. J., H. G. Rey, and R. Quian Quiroga. 2018. 'A novel and fully automatic spike-sorting implementation with variable number of features', J Neurophysiol, 120: 1859-71.

Chestek, C. A., V. Gilja, P. Nuyujukian, J. D. Foster, J. M. Fan, M. T. Kaufman, M. M. Churchland, Z. Rivera-Alvidrez, J. P. Cunningham, S. I. Ryu, and K. V. Shenoy. 2011. 'Long-term stability of neural prosthetic control signals from silicon cortical arrays in rhesus macaque motor cortex', $J$ Neural Eng, 8: 045005.

Chollet, François. 2015. "Keras." In.

Christie, B. P., D. M. Tat, Z. T. Irwin, V. Gilja, P. Nuyujukian, J. D. Foster, S. I. Ryu, K. V. Shenoy, D. E. Thompson, and C. A. Chestek. 2015. 'Comparison of spike sorting and thresholding of voltage waveforms for intracortical brain-machine interface performance', J Neural Eng, 12: 016009. 
bioRxiv preprint doi: https://doi.org/10.1101/722934; this version posted February 25, 2020. The copyright holder for this preprint (which was not certified by peer review) is the author/funder. All rights reserved. No reuse allowed without permission.

[A neural network for online spike classification] 26

651

652

653

654

655

656

657

658

659

660

661

662

663

664

665

666

667

668

669

670

671

672

673

674

675

676

677

678

679

680

681

682

683

684

685

686

687

688

689

690

691

692

693

694

695

696

697

698

699

700

Chung, J. E., J. F. Magland, A. H. Barnett, V. M. Tolosa, A. C. Tooker, K. Y. Lee, K. G. Shah, S. H. Felix, L. M. Frank, and L. F. Greengard. 2017. 'A Fully Automated Approach to Spike Sorting', Neuron, 95: 1381-94 e6.

Cohen, M. R., and A. Kohn. 2011. 'Measuring and interpreting neuronal correlations', Nat Neurosci, 14: $811-9$.

Collinger, J. L., B. Wodlinger, J. E. Downey, W. Wang, E. C. Tyler-Kabara, D. J. Weber, A. J. McMorland, M. Velliste, M. L. Boninger, and A. B. Schwartz. 2013. 'High-performance neuroprosthetic control by an individual with tetraplegia', Lancet, 381: 557-64.

Dai, J., P. Zhang, H. Sun, X. Qiao, Y. Zhao, J. Ma, S. Li, J. Zhou, and C. Wang. 2019. 'Reliability of motor and sensory neural decoding by threshold crossings for intracortical brain-machine interface', J Neural Eng, 16: 036011.

Dickey, A. S., A. Suminski, Y. Amit, and N. G. Hatsopoulos. 2009. 'Single-unit stability using chronically implanted multielectrode arrays', J Neurophysiol, 102: 1331-9.

Downey, J. E., N. Schwed, S. M. Chase, A. B. Schwartz, and J. L. Collinger. 2018. 'Intracortical recording stability in human brain-computer interface users', J Neural Eng, 15: 046016.

Flint, R. D., M. R. Scheid, Z. A. Wright, S. A. Solla, and M. W. Slutzky. 2016. 'Long-Term Stability of Motor Cortical Activity: Implications for Brain Machine Interfaces and Optimal Feedback Control', J Neurosci, 36: 3623-32.

Fraser, G. W., S. M. Chase, A. Whitford, and A. B. Schwartz. 2009. 'Control of a brain-computer interface without spike sorting', J Neural Eng, 6: 055004.

Fraser, G. W., and A. B. Schwartz. 2012. 'Recording from the same neurons chronically in motor cortex', J Neurophysiol, 107: 1970-8.

Gilja, V., P. Nuyujukian, C. A. Chestek, J. P. Cunningham, B. M. Yu, J. M. Fan, M. M. Churchland, M. T. Kaufman, J. C. Kao, S. I. Ryu, and K. V. Shenoy. 2012. 'A high-performance neural prosthesis enabled by control algorithm design', Nat Neurosci, 15: 1752-7.

Golub, M. D., B. M. Yu, and S. M. Chase. 2015. 'Internal models for interpreting neural population activity during sensorimotor control', Elife, 4.

Hochberg, L. R., D. Bacher, B. Jarosiewicz, N. Y. Masse, J. D. Simeral, J. Vogel, S. Haddadin, J. Liu, S. S. Cash, P. van der Smagt, and J. P. Donoghue. 2012. 'Reach and grasp by people with tetraplegia using a neurally controlled robotic arm', Nature, 485: 372-5.

Hochberg, L. R., M. D. Serruya, G. M. Friehs, J. A. Mukand, M. Saleh, A. H. Caplan, A. Branner, D. Chen, R. D. Penn, and J. P. Donoghue. 2006. 'Neuronal ensemble control of prosthetic devices by a human with tetraplegia', Nature, 442: 164-71.

Homer, M. L., A. V. Nurmikko, J. P. Donoghue, and L. R. Hochberg. 2013. 'Sensors and decoding for intracortical brain computer interfaces', Annu Rev Biomed Eng, 15: 383-405.

Jia, N., S. L. Brincat, A. F. Salazar-Gomez, M. Panko, F. H. Guenther, and E. K. Miller. 2017. 'Decoding of intended saccade direction in an oculomotor brain-computer interface', J Neural Eng, 14: 046007.

Kelly, R. C., M. A. Smith, J. M. Samonds, A. Kohn, A. B. Bonds, J. A. Movshon, and T. S. Lee. 2007. 'Comparison of recordings from microelectrode arrays and single electrodes in the visual cortex', J Neurosci, 27: 261-4.

Khanna, S. B., A. C. Snyder, and M. A. Smith. 2019. 'Distinct Sources of Variability Affect Eye Movement Preparation', J Neurosci, 39: 4511-26.

Kim, K. H., and S. J. Kim. 2000. 'Neural spike sorting under nearly 0-dB signal-to-noise ratio using nonlinear energy operator and artificial neural-network classifier', IEEE Trans Biomed Eng, 47: 1406-11.

Kingma, D. P., and J. Ba. 2015. "Adam: a Method for Stochastic Optimization." In International Conference on Learning Representations.

Kloosterman, F., S. P. Layton, Z. Chen, and M. A. Wilson. 2014. 'Bayesian decoding using unsorted spikes in the rat hippocampus', $J$ Neurophysiol, 111: 217-27. 
bioRxiv preprint doi: https://doi.org/10.1101/722934; this version posted February 25, 2020. The copyright holder for this preprint (which was not certified by peer review) is the author/funder. All rights reserved. No reuse allowed without permission.

[A neural network for online spike classification] 27

701

702

703

704

705

706

707

708

709

710

711

712

713

714

715

716

717

718

719

720

721

722

723

724

725

726

727

728

729

730

731

732

733

734

735

736

737

738

739

740

741

742

743

744

745

746

747

748

749

750
Kohn, A., R. Coen-Cagli, I. Kanitscheider, and A. Pouget. 2016. 'Correlations and Neuronal Population Information', Annu Rev Neurosci, 39: 237-56.

Koralek, A. C., X. Jin, J. D. Long, 2nd, R. M. Costa, and J. M. Carmena. 2012. 'Corticostriatal plasticity is necessary for learning intentional neuroprosthetic skills', Nature, 483: 331-5.

Lee, JinHyung, David Carlson, Hooshmand Shokri, Weichi Yao, Georges Goetz, Espen Hagen, Eleanor Batty, E. J. Chichilnisky, Gaute Einevoll, and Liam Paninski. 2017. 'YASS: Yet Another Spike Sorter', Advances in Neural Information Processing Systems 30 (NIPS 2017).

Lewicki, M. S. 1998. 'A review of methods for spike sorting: the detection and classification of neural action potentials', Network, 9: R53-78.

Masse, N. Y., B. Jarosiewicz, J. D. Simeral, D. Bacher, S. D. Stavisky, S. S. Cash, E. M. Oakley, E. Berhanu, E. Eskandar, G. Friehs, L. R. Hochberg, and J. P. Donoghue. 2014. 'Non-causal spike filtering improves decoding of movement intention for intracortical BCIs', $J$ Neurosci Methods, 236: 58-67.

Meyers, E. M., D. J. Freedman, G. Kreiman, E. K. Miller, and T. Poggio. 2008. 'Dynamic population coding of category information in inferior temporal and prefrontal cortex', $J$ Neurophysiol, 100: 1407-19.

Neto, J. P., G. Lopes, J. Frazao, J. Nogueira, P. Lacerda, P. Baiao, A. Aarts, A. Andrei, S. Musa, E. Fortunato, P. Barquinha, and A. R. Kampff. 2016. 'Validating silicon polytrodes with paired juxtacellular recordings: method and dataset', J Neurophysiol, 116: 892-903.

Nuyujukian, P., J. C. Kao, J. M. Fan, S. D. Stavisky, S. I. Ryu, and K. V. Shenoy. 2014. 'Performance sustaining intracortical neural prostheses', J Neural Eng, 11: 066003.

Oby, E. R., S. Perel, P. T. Sadtler, D. A. Ruff, J. L. Mischel, D. F. Montez, M. R. Cohen, A. P. Batista, and S. M. Chase. 2016. 'Extracellular voltage threshold settings can be tuned for optimal encoding of movement and stimulus parameters', J Neural Eng, 13: 036009.

Pachitariu, Marius , Nicholas Steinmetz, Shabnam Kadir, Matteo Carandini, and Kenneth Harris. 2016. 'Fast and accurate spike sorting of high-channel count probes with KiloSort', Advances in Neural Information Processing Systems 29 (NIPS 2016).

Parthasarathy, A., R. Herikstad, J. H. Bong, F. S. Medina, C. Libedinsky, and S. C. Yen. 2017. 'Mixed selectivity morphs population codes in prefrontal cortex', Nat Neurosci, 20: 1770-79.

Pedreira, Carlos, Juan Martinez, Matias J. Ison, and Rodrigo Quian Quiroga. 2012. 'How many neurons can we see with current spike sorting algorithms?', Journal of Neuroscience Methods, 211: 58-65.

Perge, J. A., M. L. Homer, W. Q. Malik, S. Cash, E. Eskandar, G. Friehs, J. P. Donoghue, and L. R. Hochberg. 2013. 'Intra-day signal instabilities affect decoding performance in an intracortical neural interface system', J Neural Eng, 10: 036004.

Perge, J. A., S. Zhang, W. Q. Malik, M. L. Homer, S. Cash, G. Friehs, E. N. Eskandar, J. P. Donoghue, and L. R. Hochberg. 2014. 'Reliability of directional information in unsorted spikes and local field potentials recorded in human motor cortex', J Neural Eng, 11: 046007.

Racz, M., C. Liber, E. Nemeth, R. B. Fiath, J. Rokai, I. Harmati, I. Ulbert, and G. Marton. 2019. 'Spike detection and sorting with deep learning', J Neural Eng.

Rey, H. G., C. Pedreira, and R. Quian Quiroga. 2015. 'Past, present and future of spike sorting techniques', Brain Res Bull, 119: 106-17.

Rizzuto, D. S., A. N. Mamelak, W. W. Sutherling, I. Fineman, and R. A. Andersen. 2005. 'Spatial selectivity in human ventrolateral prefrontal cortex', Nat Neurosci, 8: 415-7.

Rossant, C., S. N. Kadir, D. F. M. Goodman, J. Schulman, M. L. D. Hunter, A. B. Saleem, A. Grosmark, M. Belluscio, G. H. Denfield, A. S. Ecker, A. S. Tolias, S. Solomon, G. Buzsaki, M. Carandini, and K. D. Harris. 2016. 'Spike sorting for large, dense electrode arrays', Nat Neurosci, 19: 634-41.

Sadtler, P. T., K. M. Quick, M. D. Golub, S. M. Chase, S. I. Ryu, E. C. Tyler-Kabara, B. M. Yu, and A. P. Batista. 2014. 'Neural constraints on learning', Nature, 512: 423-6.

Sadtler, P. T., S. I. Ryu, E. C. Tyler-Kabara, B. M. Yu, and A. P. Batista. 2015. 'Brain-computer interface control along instructed paths', J Neural Eng, 12: 016015. 
[A neural network for online spike classification] 28

Saif-Ur-Rehman, M., R. Lienkamper, Y. Parpaley, J. Wellmer, C. Liu, B. Lee, S. Kellis, R. A. Andersen, I. Iossifidis, T. Glasmachers, and C. Klaes. 2019. 'SpikeDeeptector: A deep-learning based method for detection of neural spiking activity', J Neural Eng, 16.

Santhanam, G., M. Sahani, S. Ryu, and K. Shenoy. 2004. 'An extensible infrastructure for fully automated spike sorting during online experiments', Conf Proc IEEE Eng Med Biol Soc, 6: 4380-4.

Schafer, R. J., and T. Moore. 2011. 'Selective attention from voluntary control of neurons in prefrontal cortex', Science, 332: 1568-71.

Shoham, S., M. R. Fellows, and R. A. Normann. 2003. 'Robust, automatic spike sorting using mixtures of multivariate t-distributions', J Neurosci Methods, 127: 111-22.

Snyder, A. C., M. J. Morais, A. Kohn, and M. A. Smith. 2014. 'Correlations in V1 are reduced by stimulation outside the receptive field', J Neurosci, 34: 11222-7.

Snyder, A. C., M. J. Morais, C. M. Willis, and M. A. Smith. 2015. 'Global network influences on local functional connectivity', Nat Neurosci, 18: 736-43.

Snyder, A. C., and M. A. Smith. 2015. 'Stimulus-dependent spiking relationships with the EEG', $J$ Neurophysiol, 114: 1468-82.

Spaak, E., K. Watanabe, S. Funahashi, and M. G. Stokes. 2017. 'Stable and Dynamic Coding for Working Memory in Primate Prefrontal Cortex', J Neurosci, 37: 6503-16.

Stark, E., and M. Abeles. 2007. 'Predicting movement from multiunit activity', J Neurosci, 27: 8387-94.

Todorova, S., P. Sadtler, A. Batista, S. Chase, and V. Ventura. 2014. 'To sort or not to sort: the impact of spike-sorting on neural decoding performance', J Neural Eng, 11: 056005.

Tolias, A. S., A. S. Ecker, A. G. Siapas, A. Hoenselaar, G. A. Keliris, and N. K. Logothetis. 2007. 'Recording chronically from the same neurons in awake, behaving primates', J Neurophysiol, 98 : 3780-90.

Trautmann, E. M., S. D. Stavisky, S. Lahiri, K. C. Ames, M. T. Kaufman, D. J. O'Shea, S. Vyas, X. Sun, S. I. Ryu, S. Ganguli, and K. V. Shenoy. 2019. 'Accurate Estimation of Neural Population Dynamics without Spike Sorting', Neuron, 103: 292-308 e4.

Tremblay, S., G. Doucet, F. Pieper, A. Sachs, and J. Martinez-Trujillo. 2015. 'Single-Trial Decoding of Visual Attention from Local Field Potentials in the Primate Lateral Prefrontal Cortex Is Frequency-Dependent', J Neurosci, 35: 9038-49.

Velliste, M., S. Perel, M. C. Spalding, A. S. Whitford, and A. B. Schwartz. 2008. 'Cortical control of a prosthetic arm for self-feeding', Nature, 453: 1098-101.

Ventura, V., and S. Todorova. 2015. 'A computationally efficient method for incorporating spike waveform information into decoding algorithms', Neural Comput, 27: 1033-50.

Wang, D., Q. Zhang, Y. Li, Y. Wang, J. Zhu, S. Zhang, and X. Zheng. 2014. 'Long-term decoding stability of local field potentials from silicon arrays in primate motor cortex during a 2D center out task', J Neural Eng, 11: 036009.

Ward, M. P., P. Rajdev, C. Ellison, and P. P. Irazoqui. 2009. 'Toward a comparison of microelectrodes for acute and chronic recordings', Brain Res, 1282: 183-200.

Wood, F., M. J. Black, C. Vargas-Irwin, M. Fellows, and J. P. Donoghue. 2004. 'On the variability of manual spike sorting', IEEE Trans Biomed Eng, 51: 912-8. 
bioRxiv preprint doi: https://doi.org/10.1101/722934; this version posted February 25, 2020. The copyright holder for this preprint (which was not certified by peer review) is the author/funder. All rights reserved. No reuse allowed without permission.

[A neural network for online spike classification] 29

Figure 1. Methods of classifying waveforms: classic (manual) spike-sorting and our neural network spike classifier, Not A Sorter (NAS). (a) Spike-sorted channels with waveforms that were manually classified as spikes (green) and noise (grey). The network was trained on 24,810,795 spike-sorted neural waveforms from four monkeys. The waveforms were recorded on arrays implanted in V4 as well as U-probes placed in FEF. (b) Neural network structure and output for three sample waveforms. Each $s=52$ waveform input (I) was passed through a hidden layer (II) with $\mathrm{n}=\mathbf{5 0}$ units. The resultant linear weighting of the waveform voltages was passed through a rectified linear unit (ReLU) non-linearity (III). The output was again passed through a weighted sum (IV) followed by a sigmoid non-linearity (V). The resulting value was the network's assessment of the likelihood that the input waveform was a spike waveform (VI). We referred to this value as $P(s)$ or the "probability" of being a spike.

Figure 2. Offline decoding of planned direction from a memory guided saccade task. (a) Memory guided saccade task. The monkey fixated on a central point. A brief stimulus flash occurred followed by a delay period. When the fixation point turned off, the subject was required to make a saccade to the location of the previously flashed stimulus. (b) Decoding paradigm. The stimulus flash could occur at 8 different angles around the fixation point. A Poisson Naïve Bayes classifier was used to decode stimulus direction offline using spikes recorded from a 96-electrode Utah array in PFC $50 \mathrm{~ms}$ after target offset. For each recording session, we used 5-fold cross validation, where for each fold the data were split (step 1) into a training set to create model distributions for each direction (step 2) and an independent testing set to test the accuracy of the model's predictions (step 3). Note that the curves in step (2) do not depict actual distributions and simply represent how a Poisson decoder could use spike counts from trials in the training set to distinguish between different target conditions.

Figure 3. Classifying waveforms based on their probability of being a spike, $\mathbf{P}($ spike), by setting $\gamma$, a tunable parameter. (a-b) Waveforms classified as spikes (green) or noise (grey) for a sample channel in Monkey Pe. The network outputs a value between 0 and 1 , referred to as $P($ spike), for each waveform, where a value close to 1 means the network identifies that waveform as very likely to be a spike waveform. After running waveforms through the network, we set the minimum $P$ (spike) to classify waveforms as spikes and referred to this value as the $\gamma$ threshold. In (a) only waveforms assigned a spike probability greater than 0.20 (i.e. $\gamma$ threshold $=0.20$ ) and in $(b)$ greater than 0.70 (i.e. $\gamma$ threshold $=0.70)$ were classified as spikes. Increasing the $\gamma$ threshold by definition results in a smaller percentage of waveforms captured in the spike class. (c) Waveforms from the same channel in (a) and (b) colored based on their network assigned $P$ (spike) value. For a waveform where $\gamma_{1}<\mathbf{P}\left(\right.$ spike) $<\gamma_{2}$, the waveform would be classified as a spike when the threshold is $\gamma_{1}$, but would be labeled as noise for $\gamma_{2}$. (d) Similar to (c) except the average of waveforms across all channels within the indicated $\mathbf{P}$ (spike) ranges. Modifying the $\gamma$ threshold tuned the stringency of the spike classifications. For the channel depicted in (a)-(c) the standard deviation of the waveform noise, computed using the method described in Kelly et al. (2007), was $17.1 \mu \mathrm{V}$.

Figure 4. Using NAS spike classifications improved decoding accuracy in many sessions. (a-b) Percent decoding accuracy (black line) and percent of waveforms removed (maroon line) at different $\gamma$ thresholds for an example recording session in Monkey Pe and Wa. Chance decoding accuracy was $12.5 \%$ (verified by computing a decoding control with shuffled test trials, grey line). The decoding accuracy increased for low $\gamma$ thresholds and then reached an asymptote as $\gamma$ increased. At the highest $\gamma$ thresholds, decoding accuracy fell to chance (grey line). Other labeled relevant metrics include: decoding accuracy with threshold crossings (blue dot) maximum decoding accuracy (green dot), $\gamma$ threshold with the maximum decoding accuracy (grey dotted line), and $\Delta \%$ decoding accuracy (the difference for a given session between the decoding accuracy using the network classifications at a particular $\gamma$ threshold and the decoding accuracy with threshold crossings). (c-d) Distribution of $\gamma$ thresholds across sessions (Monkey Pe: $N=36$, Monkey Wa: $N=16$ ) that resulted in the maximum decoding accuracy for each session. (e-f) Distribution of $\Delta \%$ decoding accuracy across all sessions where the $\gamma$ threshold was the median from the distributions in (c-d). Mean $\Delta \%$ decoding accuracy shown in red. For both monkeys, using spikes classified by the network improved decoding 
accuracy on average across sessions; however, this improvement was only statistically significant for Monkey Pe (2-tailed, Wilcoxon signed rank test, Monkey Pe: $\mathbf{p}<0.0001$, Monkey Wa: $\mathbf{p}=\mathbf{0 . 0 5 7}$ ).

Figure 5. Noise on the array increased with the age of the array while decoding accuracy decreased in Monkey Pe (blue) and Monkey Wa (orange). (a) Percentage of waveforms with a P(spike) $<0.02$ over time. A waveform with a $P$ (spike) less than 0.02 was one that the network found very unlikely to be a spike. The percentage of these unlikely spike waveforms increased as the array became older (Spearman's correlation, Monkey Pe: $\varrho=0.87, p<0.0001$; Monkey Wa: $\varrho=0.90, p<0.0001$ ). (b) Percentage of waveforms with a $P($ spike $)>0.70$ over time. The percentage of waveforms that the network found to be strongly spike-like decreased as the array became older (Spearman's correlation, Monkey Pe: $0=-0.87, p<0.0001$; Monkey Wa: $\mathrm{o}=\mathbf{- 0 . 9 4}, \mathrm{p}<0.0001)$. (c) Decoding accuracy with threshold crossings (i.e. $\gamma=0)$ decreased as the array aged (Spearman's correlation, Monkey Pe: $\mathbf{0}=\mathbf{- 0 . 8 6}, \mathrm{p}<\mathbf{0 . 0 0 0 1}$; Monkey Wa: $\mathrm{o}=\mathbf{- 0 . 6 9 ,} \mathrm{p}=\mathbf{0 . 0 0 4}$ ). (d) Change in percent decoding accuracy using network-classified spikes relative to decoding accuracy with threshold crossings ( $\Delta \%$ decoding accuracy). We computed a distribution of the maximum $\gamma$ threshold (similar to Fig. 4c-d) for sessions that were 0-50 days post array implant and used the median to set the $\gamma$ threshold before computing decoding accuracy for those sessions. We repeated this for sessions more than $\mathbf{5 0}$ days post array implant. In Monkey Pe, using NAS classifications improved decoding accuracy (2-tailed, Wilcoxon signed rank test, $p<0.0001)$ and in Monkey Wa, using the classifications neither hurt nor helped decoding significantly $(p=0.09)$. In both subjects, the network helped decoding more in the late array sessions ( $>50$ days post implant) compared to the early sessions (2-tailed, Wilcoxon rank sum, Monkey Pe: $p=0.01$; Monkey Wa: $\mathbf{p}=0.01$ ). (e) Mean normalized decoding accuracy across early sessions (open circles) and late sessions (filled circles) as a function of $\gamma$ threshold. Shading represents +/- 1 SEM. Using the network classifications at any $\gamma$ threshold greater than zero was more helpful for late sessions than it was for early sessions.

Figure 6. Using NAS classifications was comparable to using spike-sorted data for decoding. $\Delta \%$ decoding accuracy was calculated for both NAS classifications and spike-sorted waveforms as the change from decoding accuracy with threshold crossings. Top: Distribution of $\Delta \%$ decoding accuracy using NAS classifications aggregated across subjects ( $\gamma$ threshold selected as in 5d). Using NAS classifications improved decoding accuracy from that with threshold crossings (2-tailed, Wilcoxon signed rank test, $p<0.0001$ ). Right: Distribution of $\Delta \%$ decoding accuracy using manual spike-sorting aggregated across subjects. Using spikesorted classifications also improved decoding accuracy from that with threshold crossings (2-tailed, Wilcoxon signed rank test, $p<0.0001)$. Center: The joint distribution of $\Delta \%$ decoding accuracy with spike-sorting and NAS classified spikes. Using our network's classifications was at least as helpful as spike-sorting for decoding (paired, 2-tailed, Wilcoxon signed rank test, Monkey Pe: $p=0.75$; Monkey Wa: $p=0.09$ ). 
(a) Channels sorted via classic spike-sorting
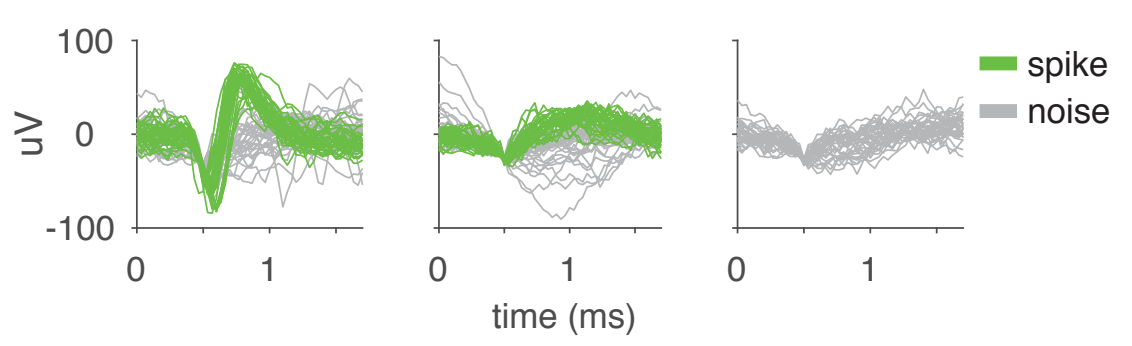

(b)

I. waveform

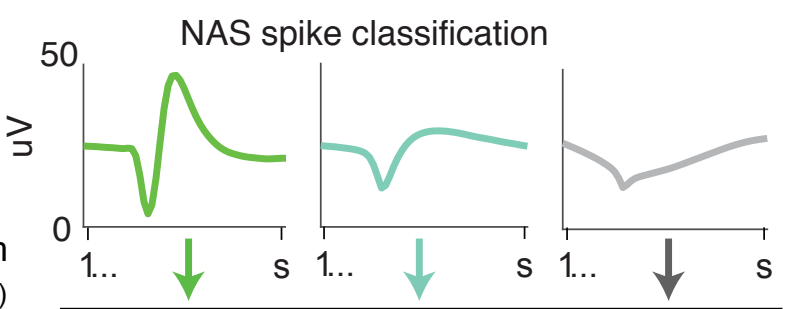

input $(1 \times S)$

$\begin{array}{ll}\text { II. } & \text { hidden } \\ \text { layer }(1 \times n)\end{array}$

III. ReLU

IV. $\begin{aligned} & \text { output } \\ & \text { neuron }\end{aligned}$

sigmoid
function

VI. probability of being a spike

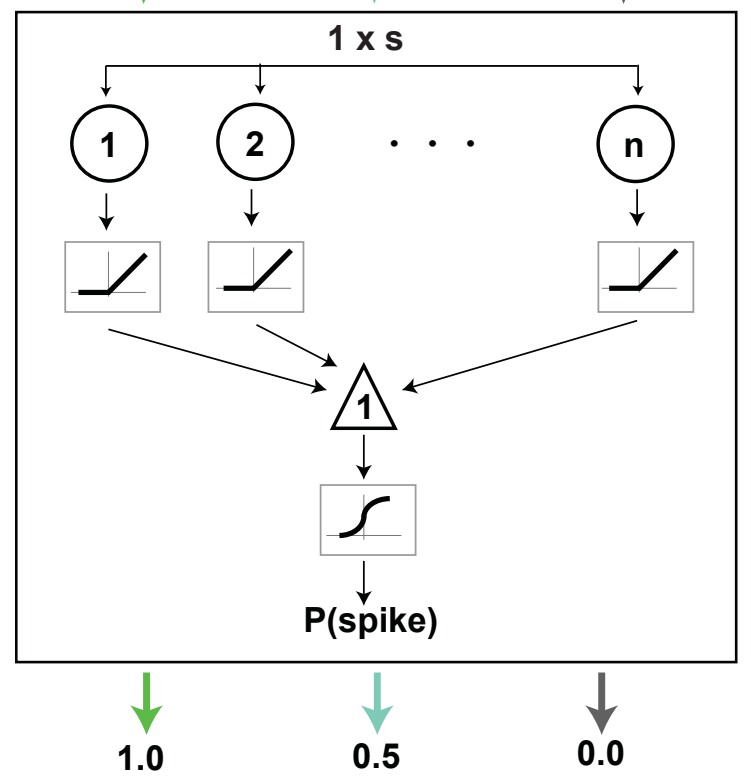


(a) Memory guided saccade task

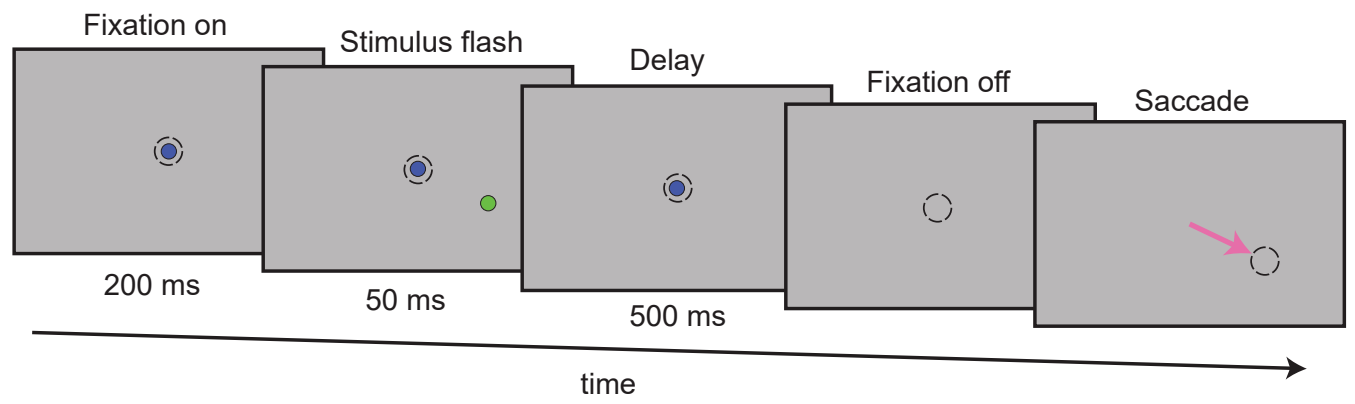

(b) Decoding paradigm
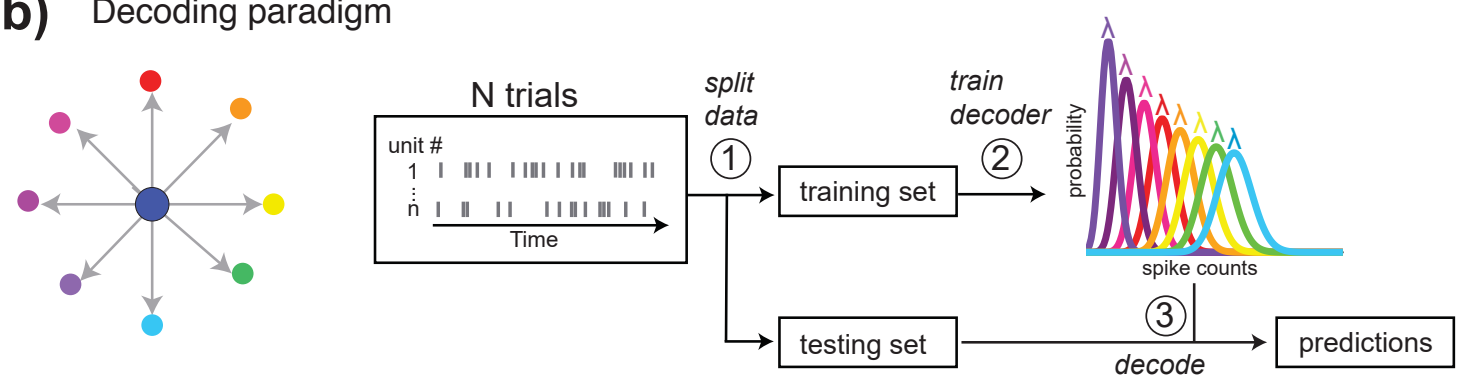


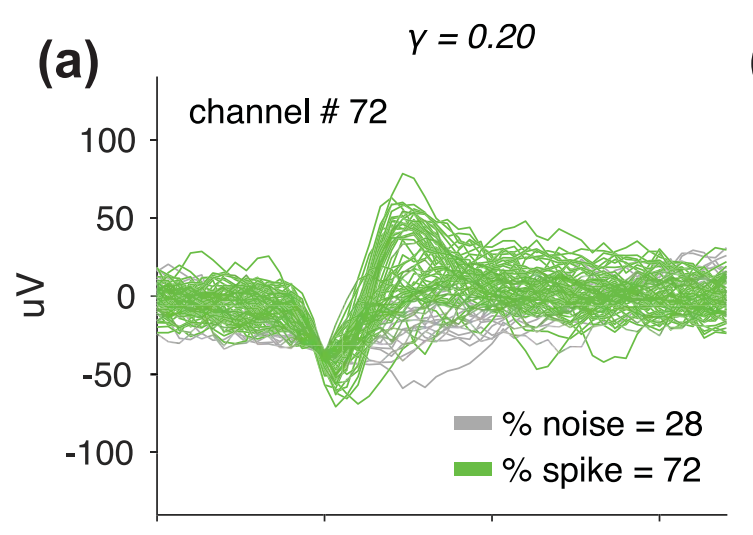

(b)

$$
\gamma=0.70
$$

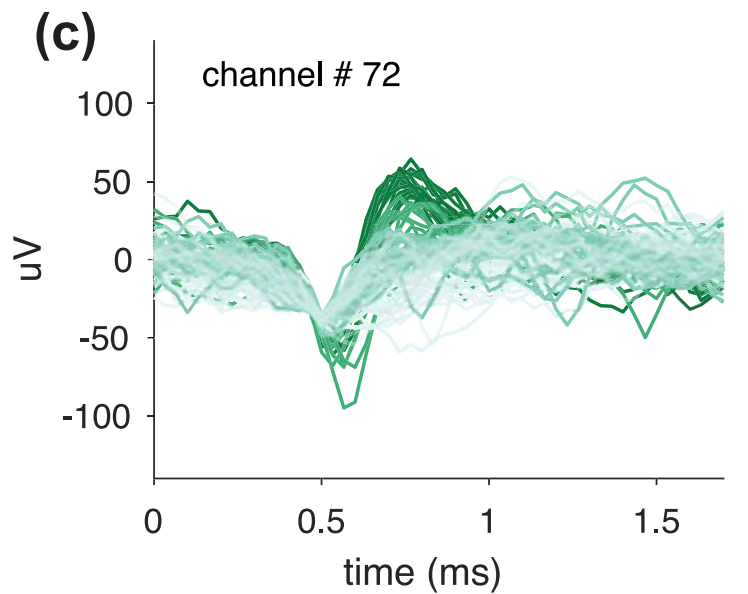

(d)

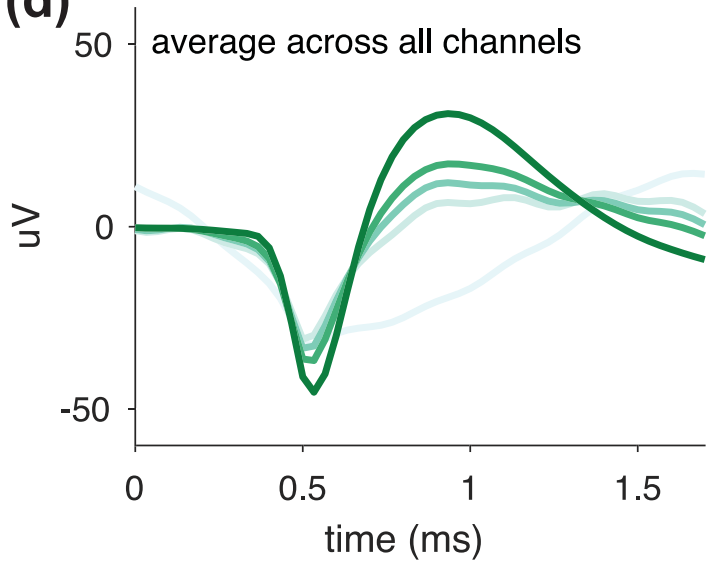

$$
\begin{array}{lll}
0>\mathrm{P}(\text { spike })>0.06 & 0.20>\mathrm{P}(\text { spike })>0.50 \\
0.06>\mathrm{P}(\text { spike })>0.20 & 0.50>\mathrm{P}(\text { spike })>0.70
\end{array} \quad 0.70>\mathrm{P}(\text { spike })>1
$$


(a)

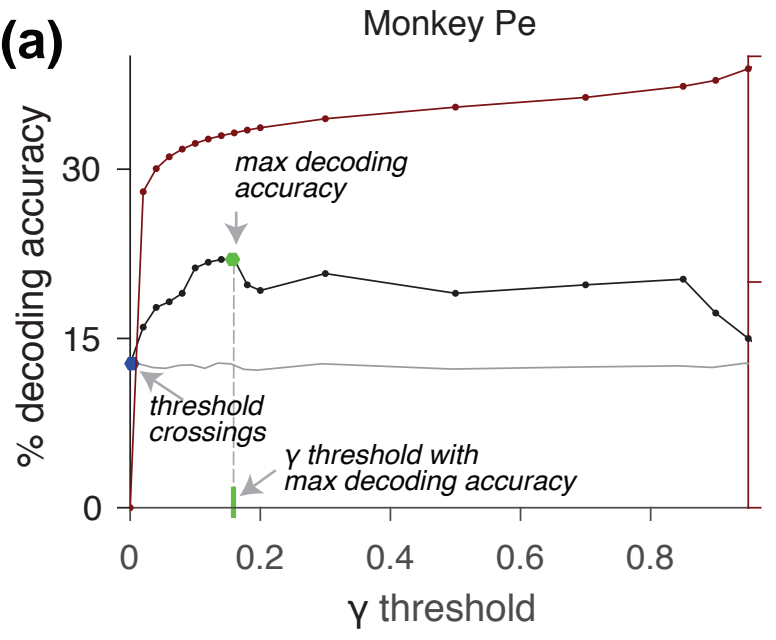

(c)

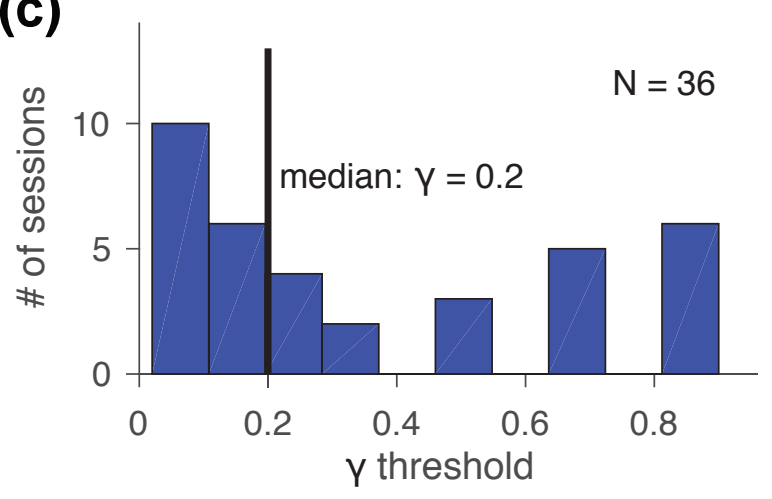

(b)

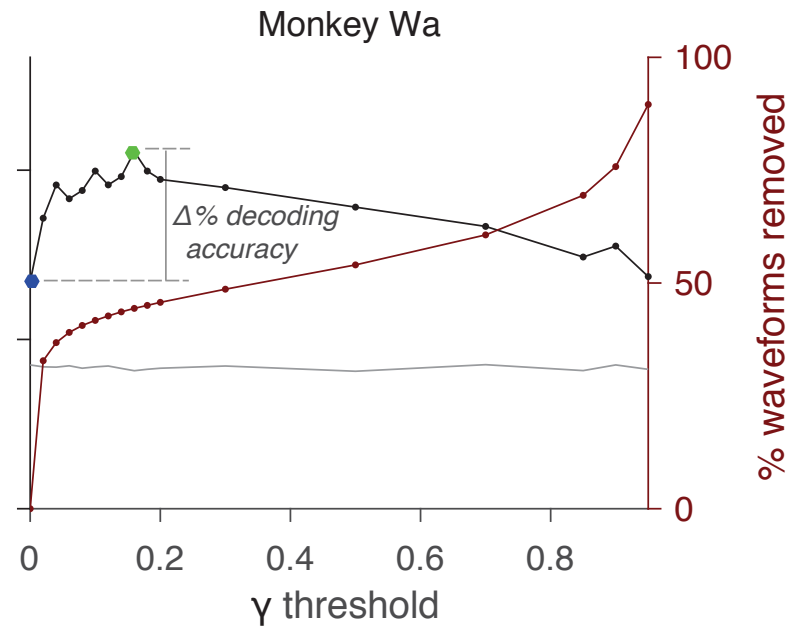

(d)

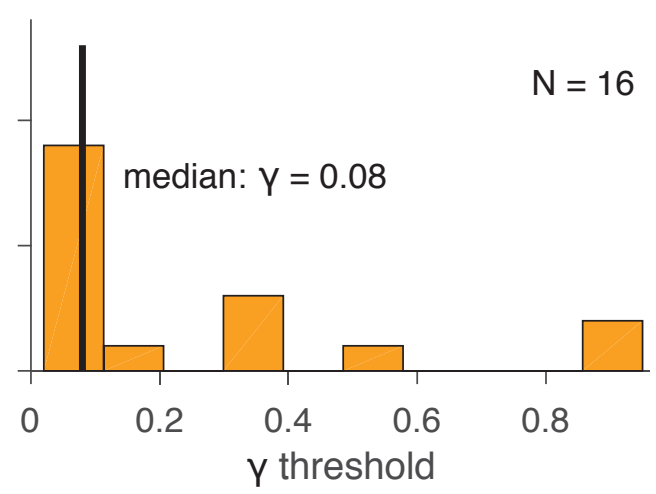

(e)

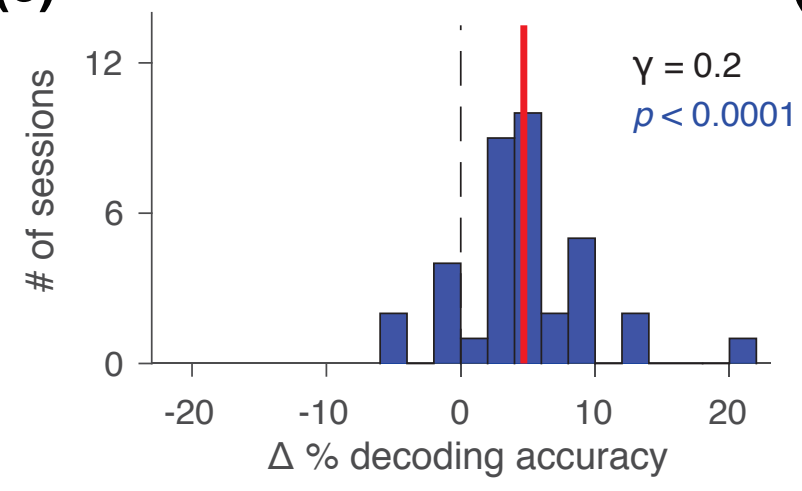

(f)

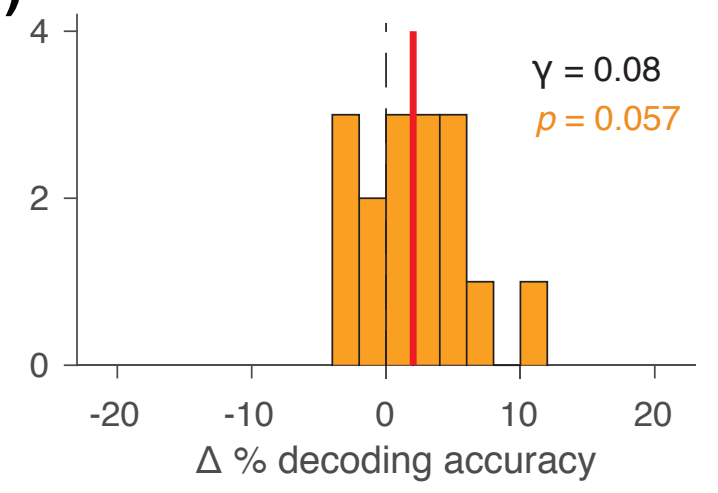




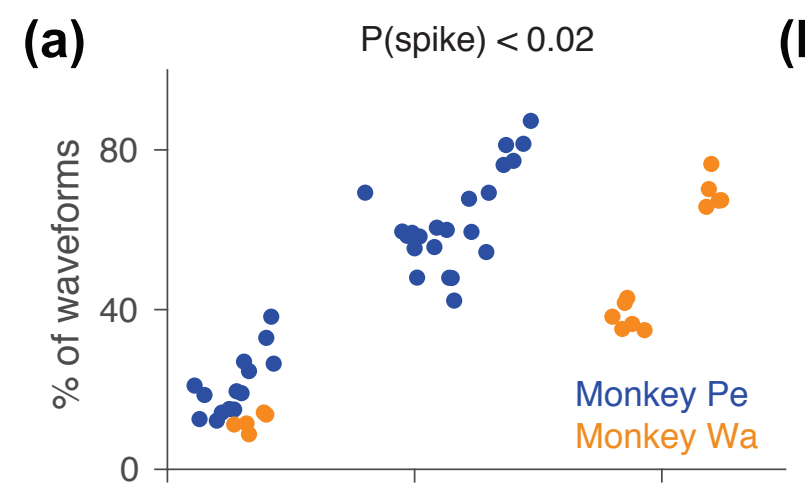

(b)
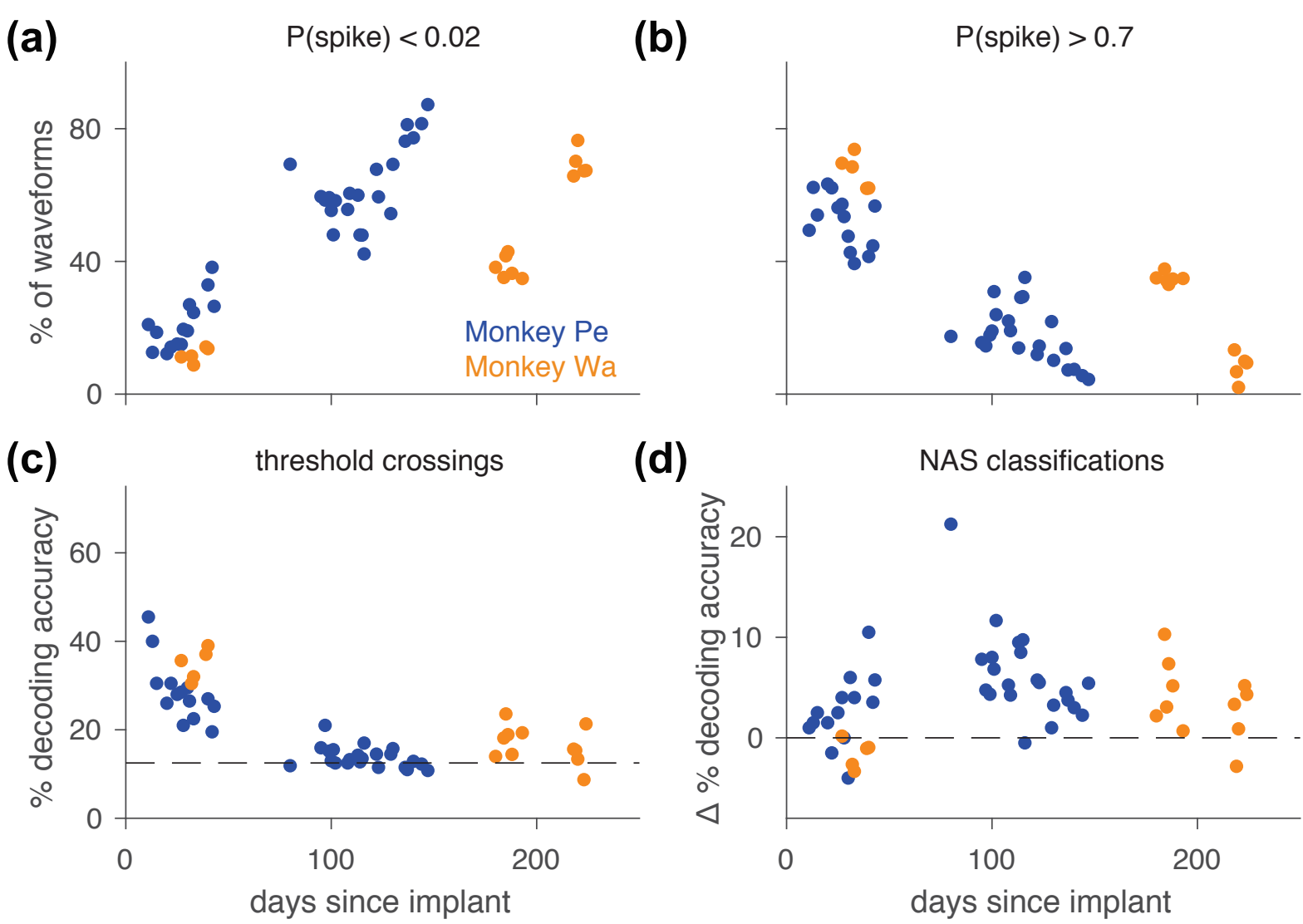

(d)

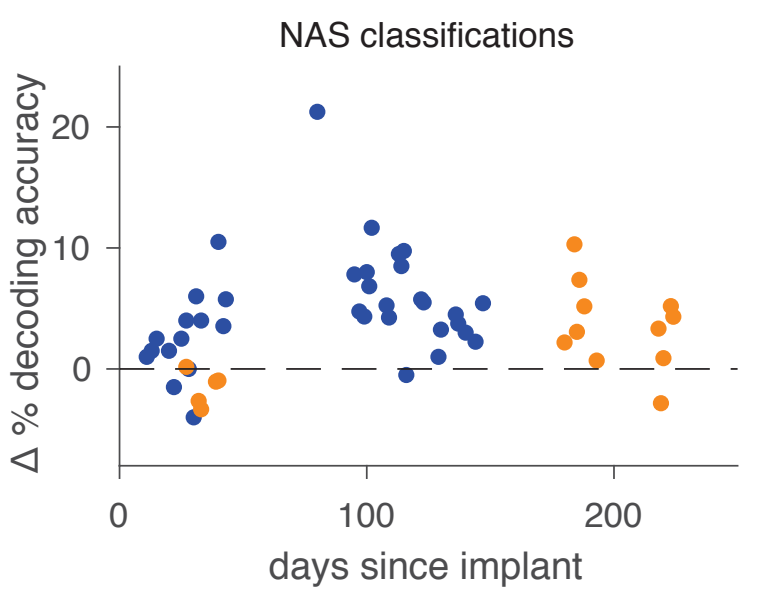

(e)

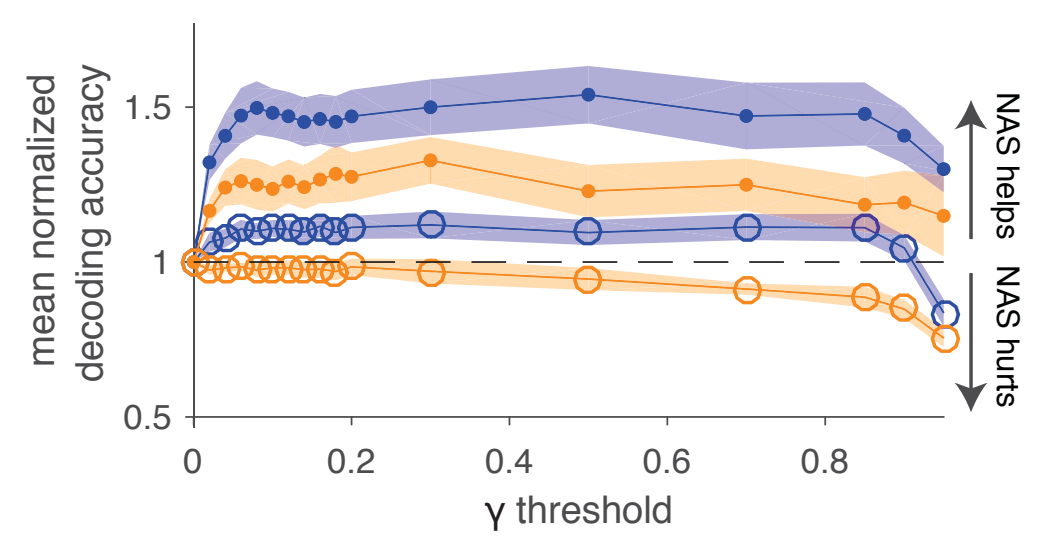

- Monkey Pe 0-50 days post-implant Monkey Wa 0-50 days post-implant

- Monkey Pe $>50$ days post-implant Monkey Wa $>50$ days post-implant 


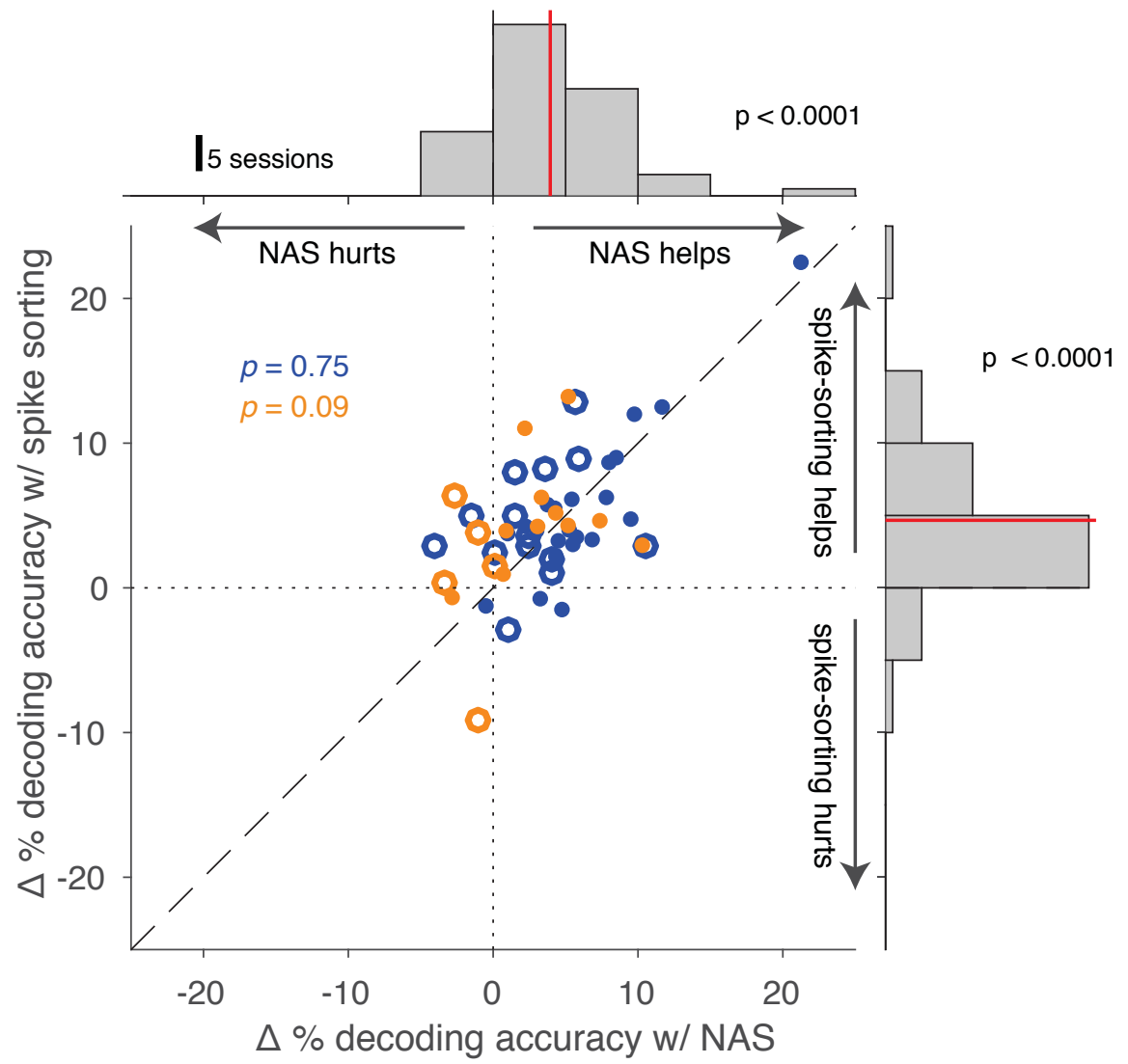

- Monkey Pe 0-50 days post-implant 1 Monkey Wa 0-50 days post-implant

- Monkey $\mathrm{Pe}>50$ days post-implant Monkey Wa $>50$ days post-implant 